\title{
A CONVERGENT ADAPTIVE FINITE ELEMENT METHOD FOR ELLIPTIC DIRICHLET BOUNDARY CONTROL PROBLEMS
}

\author{
WEI GONG *, WENBIN LIU ${ }^{\dagger}$, ZHIYU TAN $^{\diamond}$, AND NINGNING YAN ${ }^{\square}$
}

\begin{abstract}
This paper concerns the adaptive finite element method for elliptic Dirichlet boundary control problems in energy space. The contribution of this paper is twofold. Firstly, we rigorously derive efficient and reliable a posteriori error estimates for finite element approximations of the Dirichlet boundary control problems. As a byproduct, a priori error estimates can be derived in a simple way by introducing appropriate auxiliary problems and establishing certain norm equivalence. Secondly, for the coupled elliptic partial differential system involving the control, the state and the adjoint state which resulted from the first order optimality system, we prove that the sequence of adaptively generated discrete solutions, guided by our newly derived a posteriori error indicators, converge to the true solutions along with the convergence of the error estimators. We give some numerical results to confirm our theoretical findings.
\end{abstract}

Keywords: optimal control problem, elliptic equation, Dirichlet bounary control, energy space, adaptive finite element method, convergence

Subject Classification: 49J20, 65K10, 65N12, 65N15, 65N30.

\section{INTRODUCTION}

In this paper we consider the following elliptic Dirichlet boundary control problem:

$$
\min _{u \in H^{1}(\Omega)} J(y, u)=\frac{1}{2}\left\|y-y^{d}\right\|_{0, \Omega}^{2}+\frac{\alpha}{2}\|\nabla u\|_{0, \Omega}^{2}
$$

subject to

$$
\left\{\begin{aligned}
-\Delta y=f & \text { in } \Omega \\
y=u & \text { on } \Gamma:=\partial \Omega
\end{aligned}\right.
$$

where $\alpha>0$ is the penalty parameter.

There are different types of objective functionals for Dirichlet boundary control problems, depending on the choice of the control space. The most popular one is looking for the optimal control in $L^{2}(\Gamma)$ as follows:

$$
\min _{u \in L^{2}(\Gamma)} J(y, u)=\frac{1}{2}\left\|y-y^{d}\right\|_{0, \Omega}^{2}+\frac{\alpha}{2}\|u\|_{0, \Gamma}^{2} \cdot
$$

In this case the governing state equation (1.2) has to be understood in the very weak sense, since the inhomogeneous Dirichlet boundary condition for elliptic equation is only in $L^{2}(\Gamma)$. This formulation is easy to implement numerically and usually results in optimal controls with low regularity. There are extensive numerical studies for elliptic Dirichlet boundary control problems based on this formulation, we refer to [1.4.9.28] for the a priori error estimates. In [12] this formulation is extended to study parabolic Dirichlet boundary control problems. With the choice of $L^{2}(\Gamma)$ as control space, we should also mention [13] for the numerical scheme based on mixed variational scheme and [5] for the Robin penalization which transforms the Dirichlet control problem into a Robin control problem.

* NCMIS, LSEC, Institute of Computational Mathematics, Academy of Mathematics and Systems Science, Chinese Academy of Sciences, Beijing 100190, China. Email: gongwei@amss.ac.cn.

$\dagger$ KBS, University of Kent, Kent CT2 7PE, UK. Email: W.B.Liu@kent.ac.uk.

${ }^{\circ}$ Department of Mathematics, Hong Kong Baptist University, Hong Kong, China. Email: tanzhiyu@amss . ac. cn.

$\square$ NCMIS, LSEC, Institute of Systems Science, Academy of Mathematics and Systems Science, Chinese Academy of Sciences, Beijing 100190, China. Email: ynn@amss.ac.cn. 
The second approach is to find optimal controls in the energy space, i.e., $H^{\frac{1}{2}}(\Gamma)$, that is

OCP_energy

$$
\min _{u \in H^{\frac{1}{2}(\Gamma)}} J(y, u)=\frac{1}{2}\left\|y-y^{d}\right\|_{0, \Omega}^{2}+\frac{\alpha}{2}|u|_{H^{\frac{1}{2}(\Gamma)}}^{2} .
$$

We refer to [31] for this approach where pointwise control constraints of box type are also imposed. With this choice of control space one can define the standard weak solution for the state equation (1.2). However, we have to resort to the Steklov-Poincare operator to derive the optimality condition on the boundary, which may cause some difficulties in numerical implementation.

Note that we have an equivalent form of the norm in $H^{\frac{1}{2}}(\Gamma)$ :

$$
\|u\|_{H^{\frac{1}{2}(\Gamma)}}=\min _{y \in H^{1}(\Omega):\left.y\right|_{\Gamma}=u}\|y\|_{1, \Omega} .
$$

This motivates us to define the semi-norm in $H^{\frac{1}{2}}(\Gamma)$ as follows:

$$
|u|_{H^{\frac{1}{2}(\Gamma)}}=\min _{y \in H^{1}(\Omega):\left.y\right|_{\Gamma}=u}\|\nabla y\|_{0, \Omega} .
$$

It is well-known that there exists the harmonic extension $y_{u} \in H^{1}(\Omega)$ for any $u \in H^{\frac{1}{2}}(\Gamma)$ satisfying

harmonic

$$
\left\{\begin{aligned}
-\Delta y_{u}=0 & \text { in } \Omega, \\
y_{u}=u & \text { on } \Gamma .
\end{aligned}\right.
$$

Therefore, we are led to an equivalent definition of the $H^{\frac{1}{2}}(\Gamma)$ semi-norm

$$
|u|_{H^{\frac{1}{2}(\Gamma)}}=\left\|\nabla y_{u}\right\|_{0, \Omega} .
$$

This leads to the penalization of the control in $H^{1}(\Omega)$ as 1.1 . This modified scheme for elliptic Dirichlet boundary control problem was first studied in [6]. The advantage of the Dirichlet boundary control problem in energy space lies in that we do not need to impose convexity assumption on the domain when we study the well-posedness of the problem and intend to derive a priori and a posteriori error estimates.

It is well-known that the solution of Dirichlet boundary control problems usually exhibits low regularity (see, e.g., [4]]). Thus, the well-developed adaptive finite element method provides the possibility to enhance the approximation accuracy by less computational cost. But so far we do not aware of any work on adaptive finite element method to solve Dirichlet boundary control problems, except the attempt in [6], possibly due to the specifically chosen variational formulations. For instance, if we use the first approach (1.3) to study Dirichlet boundary control problem, the mismatch between the $H^{1}$-norm and the $L^{2}$-norm on the boundary for discrete finite element functions introduces the inverse estimate which may cause difficulty when we intend to derive a posteriori error estimate. In [6] the authors attempted to derive a posteriori error estimate, however, the proof contains some flaws. In this paper we intend to give a rigorous proof.

The contribution of this paper is twofold. Firstly, we rigorously derive efficient and reliable a posteriori error estimates for finite element approximations of the Dirichlet boundary control problems. As a byproduct, a priori error estimates can be derived in a simple way by introducing appropriate auxiliary problems and establishing certain norm equivalence. Secondly, for the coupled elliptic partial differential system involving the control, the state and the adjoint state which resulted from the first order optimality system, we prove that the sequence of adaptively generated discrete solutions, guided by our newly derived a posteriori error indicators, converge to the true solutions along with the convergence of the error estimators.

We note that with the new error analysis the results can be generalized to three dimensional case and more general governing state equations trivially. We also note that the first order optimality system of the Dirichlet boundary control problem in energy space can be viewed as a strongly coupled partial differential system. Thus, the techniques developed in current paper can be generalized to prove the convergence of AFEM for such kind of coupled partial differential equations. However, at this moment we can not prove the error reduction property and optimality of the adaptive algorithm, as done in [10,29] for elliptic boundary value problems and [14-16] for elliptic optimal control problems with distributed control, due to the lack of (quasi-)orthogonality of the strongly coupled elliptic system. For the similar plain convergence of 
adaptive algorithm for elliptic distributed control problem we refer to [20], and to [34] and [35] for parameter identification problems which share some similarities with PDE-constrained optimal control problems. The proof of plain convergence of adaptive algorithm is based on the techniques developed in [30] and [33].

The remaining of this paper is organized as follows: In Section 2 we recall the formulation of the Dirichlet boundary control problems in energy space, and give some important observations which will play crucial role in following error analysis. A priori error estimate is derived with newly developed techniques compared to [6]. In Section 3 we derive efficient and reliable a posteriori error estimates for finite element approximations of the Dirichlet boundary control problems by introducing appropriate auxiliary problems. Section 4 is devoted to convergence analysis of the adaptive algorithm. At last, In Section 5 we carry out some numerical experiments to confirm our theoretical findings.

Let $\Omega \subset \mathbb{R}^{2}$ be a bounded polygonal domain which is not necessarily convex. We denote by $W^{m, q}(\Omega)$ the usual Sobolev space of order $m \geq 0,1 \leq q<\infty$ with norm $\|\cdot\|_{m, q, \Omega}$ and seminorm $|\cdot|_{m, q, \Omega}$. For $q=2$ we denote $W^{m, q}(\Omega)$ by $H^{m}(\Omega)$ and $\|\cdot\|_{m, \Omega}=\|\cdot\|_{m, 2, \Omega}$, which is a Hilbert space. Note that $H^{0}(\Omega)=L^{2}(\Omega)$ and $H_{0}^{1}(\Omega)=\left\{v \in H^{1}(\Omega): v=0\right.$ on $\left.\partial \Omega\right\}$. We denote $C$ a generic positive constant which may stand for different values at its different occurrences but does not depend on mesh size. We use the symbol $A \lesssim B$ to denote $A \leq C B$ for some constant $C$ that is independent of mesh size. We write $A \approx B$ if $A \lesssim B$ and $B \lesssim A$.

\section{OPTIMAL CONTROL PROBLEM AND ITS FINITE ELEMENT APPROXIMATION}

The weak formulation of 1.2 can be stated as: Given $u \in H^{1}(\Omega)$, find $y \in H^{1}(\Omega)$ such that $\left.y\right|_{\partial \Omega}=u$ and

$$
a(y, w)=(f, w) \quad \forall w \in H_{0}^{1}(\Omega) .
$$

By invoking the harmonic extension of $u$ we can define an alternative weak formulation: Let $y=y^{f}+u$ such that $y^{f} \in H_{0}^{1}(\Omega)$ and

$$
a\left(y^{f}, w\right)=(f, w)-a(u, w) \quad \forall w \in H_{0}^{1}(\Omega) .
$$

We may introduce the solution operator $G: L^{2}(\Omega) \times H^{1}(\Omega) \rightarrow H_{0}^{1}(\Omega)$ associated with 2.2 such that $y^{f}=G(f, u)$. Therefore, we can introduce the solution operator for the state equation 1.2 as $S: L^{2}(\Omega) \times$ $H^{1}(\Omega) \rightarrow H^{1}(\Omega)$ with $y:=S(f, u)=G(f, u)+u$. Then we are led to a reduced optimization problem

$$
\min _{u \in H^{1}(\Omega)} \hat{J}(u)=J(S(f, u), u) .
$$

Note that $\frac{\alpha}{2}\|\nabla u\|_{0, \Omega}^{2}$ is not necessarily coercive and strictly convex in $H^{1}(\Omega)$ since $\|\nabla u\|_{0, \Omega}$ is not a norm. However, due to the dependence on $u$ of $y$ through the state equation we can conclude that $\hat{J}(u)$ is coercive in $H^{1}(\Omega)$ and also strictly convex. By using standard arguments (see for instance [22]) we can prove that the above reduced optimization problem admits a unique solution.

Similar to [6] we can derive the first order optimality condition for the optimal control problem [1.1 1.2 as follows: there exists $\left(u, y^{f}, p\right) \in H^{1}(\Omega) \times H_{0}^{1}(\Omega) \times H_{0}^{1}(\Omega)$ such that

OCP_OPT

$$
\left\{\begin{array}{l}
a\left(y^{f}, w\right)=(f, w)-a(u, w) \quad \forall w \in H_{0}^{1}(\Omega) ; \\
a(w, p)=\left(y-y^{d}, w\right) \quad \forall w \in H_{0}^{1}(\Omega) ; \\
\alpha a(u, v)=a(v, p)+\left(y^{d}-y, v\right) \quad \forall v \in H^{1}(\Omega),
\end{array}\right.
$$

where $y=y^{f}+u \in H^{1}(\Omega)$. The adjoint state equation and the control equation can be written as

adjoint

$$
\left\{\begin{array}{cc}
-\Delta p=y-y^{d} & \text { in } \Omega, \\
p=0 & \text { on } \Gamma
\end{array}\right.
$$

and

control

$$
\left\{\begin{array}{cc}
-\Delta u=0 & \text { in } \Omega, \\
\alpha \frac{\partial u}{\partial n}=\frac{\partial p}{\partial n} & \text { on } \Gamma
\end{array}\right.
$$

in the sense of distribution. It follows from the second and the third equations in 2.4) that $u$ is harmonic in the sense that

$$
a(u, v)=0 \quad \forall v \in H_{0}^{1}(\Omega) .
$$


Therefore, $u=S(0, u)$ and the first equation in 2.4$)$ can be written as

$$
a\left(y^{f}, w\right)=(f, w) \quad \forall w \in H_{0}^{1}(\Omega) .
$$

It is clear that $y^{f}$ can be decoupled and independent on $u$. Moreover, we can conclude from $(2.4)$ and $(2.5)$ that $\int_{\Gamma} \frac{\partial p}{\partial n} d s=0$, which ensures the well posedness of the control equation as a pure Neumann problem. Note that the third equation in 2.4 can be written as

$$
\alpha a(u, v)+(u, v)=a(v, p)+\left(y^{d}-y^{f}, v\right) \quad \forall v \in H^{1}(\Omega)
$$

so the well-posedness of the control equation for given $p$ and $y^{f}$ can be proved by Lax-Milgram theorem. This observation is very important in our following error analysis.

Remark 2.1. We remark that the above formulation can be easily extended to a general second order elliptic equation

$$
-\sum_{i, j=1}^{2} \partial_{x_{j}}\left(a_{i j} \partial_{x_{i}} y\right)+a_{0} y=f \quad \text { in } \Omega ; \quad y=u \quad \text { on } \Gamma \text {. }
$$

Here $0 \leq a_{0}<\infty, a_{i j} \in W^{1, \infty}(\Omega)(i, j=1,2)$ and $\left(a_{i j}\right)_{2 \times 2}$ is symmetric and positive definite. Let

$$
a(y, v)=\int_{\Omega}\left(\sum_{i, j=1}^{2} a_{i j} \frac{\partial y}{\partial x_{i}} \frac{\partial v}{\partial x_{j}}+a_{0} y v\right) d x .
$$

The corresponding Dirichlet boundary control problem in energy space can be formulated as

$$
\min _{u \in H^{1}(\Omega)} J(y, u)=\frac{1}{2}\left\|y-y^{d}\right\|_{0, \Omega}^{2}+\frac{\alpha}{2}|u|_{a, \Omega}^{2},
$$

where $|\cdot|_{a, \Omega}=\sqrt{a(\cdot, \cdot)}$.

Next, let us consider the finite element approximation of 1.1 . Let $\mathcal{T}_{h}$ be a regular triangulation of $\Omega$ such that $\bar{\Omega}=\cup_{\tau \in \mathcal{T}_{h}} \bar{\tau}$. In this paper, we use $\mathcal{E}_{h}^{i}$ to denote the set of interior edges of $\mathcal{T}_{h}$ and denote $\mathcal{E}_{h}^{b}$ the set of boundary edges. On $\mathcal{T}_{h}$ we construct the piecewise linear and continuous finite element space $V_{h}$ such that $V_{h} \subset C(\bar{\Omega})$ and set $V_{h}^{0}:=V_{h} \cap H_{0}^{1}(\Omega)$.

Now we consider the finite element approximation of the control problem (1.1)- 1.2$]$ :

OCP_h

OCP_state_h

$$
\min _{u_{h} \in V_{h}} J\left(y_{h}, u_{h}\right)=\frac{1}{2}\left\|y_{h}-y^{d}\right\|_{0, \Omega}^{2}+\frac{\alpha}{2}\left\|\nabla u_{h}\right\|_{0, \Omega}^{2}
$$

subject to

$$
\left\{\begin{array}{c}
a\left(y_{h}, w_{h}\right)=\left(f, w_{h}\right) \quad \forall w \in V_{h}^{0} ; \\
\left.y_{h}\right|_{\partial \Omega}=u_{h} .
\end{array}\right.
$$

Similarly, we can define the discrete solution operator $G_{h}: L^{2}(\Omega) \times H^{1}(\Omega) \rightarrow V_{h}^{0}$ such that for any $u_{h} \in V_{h}$, $y_{h}^{f}:=G_{h}\left(f, u_{h}\right) \in V_{h}^{0}$ satisfies

$$
a\left(y_{h}^{f}, w_{h}\right)=\left(f, w_{h}\right)-a\left(u_{h}, w_{h}\right) \quad \forall w_{h} \in V_{h}^{0} .
$$

We also define $S_{h}: L^{2}(\Omega) \times H^{1}(\Omega) \rightarrow V^{h}$ so that we can write $y_{h}:=S_{h}\left(f, u_{h}\right)=G_{h}\left(f, u_{h}\right)+u_{h}$. The first order optimality system for the discrete optimal control problem 2.9$]-2.10$ is as follows: Find $\left(u_{h}, y_{h}^{f}, p_{h}\right) \in$ $V_{h} \times V_{h}^{0} \times V_{h}^{0}$ such that

$$
\left\{\begin{array}{l}
a\left(y_{h}^{f}, w_{h}\right)=\left(f, w_{h}\right)-a\left(u_{h}, w_{h}\right) \quad \forall w_{h} \in V_{h}^{0} ; \\
a\left(w_{h}, p_{h}\right)=\left(y_{h}-y^{d}, w_{h}\right) \quad \forall w_{h} \in V_{h}^{0} ; \\
\alpha a\left(u_{h}, v_{h}\right)=a\left(v_{h}, p_{h}\right)+\left(y^{d}-y_{h}, v_{h}\right) \quad \forall v \in V_{h},
\end{array}\right.
$$

where $y_{h}=y_{h}^{f}+u_{h} \in V_{h}$. Since the state equation is self-adjoint we may write $p_{h}=G_{h}\left(y_{h}-y^{d}, 0\right)$. Similarly, we can derive that

$$
a\left(u_{h}, w_{h}\right)=0 \quad \forall w_{h} \in V_{h}^{0}
$$


Therefore, $u_{h}=S_{h}\left(0, u_{h}\right)$ and the first equation in 2.12) can be written as

$$
a\left(y_{h}^{f}, w_{h}\right)=\left(f, w_{h}\right) \quad \forall w_{h} \in V_{h}^{0} .
$$

Similar to 2.8) we have

control_dis

Lm:norm_equi

norm_equi_con

norm_equi_dis

$$
\alpha a\left(u_{h}, v_{h}\right)+\left(u_{h}, v_{h}\right)=a\left(v_{h}, p_{h}\right)+\left(y^{d}-y_{h}^{f}, v_{h}\right) \quad \forall v_{h} \in V_{h} .
$$

The following norm equivalence property plays very important role in our error analysis.

Lemma 2.2. We have the following norm equivalence property: For any $v \in H^{1}(\Omega)$ and $v_{h} \in V_{h}$ there hold

$$
\begin{array}{r}
\|S(0, v)\|_{0, \Omega}^{2}+\alpha\|\nabla v\|_{0, \Omega}^{2} \approx\|v\|_{1, \Omega}^{2}, \\
\left\|S_{h}\left(0, v_{h}\right)\right\|_{0, \Omega}^{2}+\alpha\left\|\nabla v_{h}\right\|_{0, \Omega}^{2} \approx\left\|v_{h}\right\|_{1, \Omega}^{2} .
\end{array}
$$

Proof. For any $v \in H^{1}(\Omega)$ we have

$$
\begin{aligned}
\|v\|_{1, \Omega}^{2} & =\|S(0, v)-G(0, v)\|_{1, \Omega}^{2} \leq 2\|S(0, v)\|_{1, \Omega}^{2}+2\|G(0, v)\|_{1, \Omega}^{2} \\
& \leq 2\|S(0, v)\|_{0, \Omega}^{2}+2\|\nabla S(0, v)\|_{0, \Omega}^{2}+2\|G(0, v)\|_{1, \Omega}^{2} \\
& \leq 2\|S(0, v)\|_{0, \Omega}^{2}+4\|\nabla G(0, v)\|_{0, \Omega}^{2}+4\|\nabla v\|_{0, \Omega}^{2}+2\|G(0, v)\|_{1, \Omega}^{2} \\
& \leq C\left(\|S(0, v)\|_{0, \Omega}^{2}+\|\nabla v\|_{0, \Omega}^{2}\right) \\
& \leq C\left(\|S(0, v)\|_{0, \Omega}^{2}+\alpha\|\nabla v\|_{0, \Omega}^{2}\right)
\end{aligned}
$$

and

$$
\begin{aligned}
\|S(0, v)\|_{0, \Omega}^{2}+\alpha\|\nabla v\|_{0, \Omega}^{2} & \leq 2\|G(0, v)\|_{0, \Omega}^{2}+2\|v\|_{0, \Omega}^{2}+\alpha\|\nabla v\|_{0, \Omega}^{2} \\
& \leq C\left(\|v\|_{0, \Omega}^{2}+\alpha\|\nabla v\|_{0, \Omega}^{2}\right) \\
& \leq C\|v\|_{1, \Omega}^{2},
\end{aligned}
$$

where $S(0, v)=G(0, v)+v$. Therefore, $\|S(0, v)\|_{0, \Omega}^{2}+\alpha\|\nabla v\|_{0, \Omega}^{2} \approx\|v\|_{1, \Omega}^{2}$.

Similarly, for any $v_{h} \in V_{h}$ it follows that

$$
\begin{aligned}
\left\|v_{h}\right\|_{1, \Omega}^{2} & =\left\|S_{h}\left(0, v_{h}\right)-G_{h}\left(0, v_{h}\right)\right\|_{1, \Omega}^{2} \leq 2\left\|S_{h}\left(0, v_{h}\right)\right\|_{1, \Omega}^{2}+2\left\|G_{h}\left(0, v_{h}\right)\right\|_{1, \Omega}^{2} \\
& \leq 2\left\|S_{h}\left(0, v_{h}\right)\right\|_{0, \Omega}^{2}+2\left\|\nabla S_{h}\left(0, v_{h}\right)\right\|_{0, \Omega}^{2}+2\left\|G_{h}\left(0, v_{h}\right)\right\|_{1, \Omega}^{2} \\
& \leq 2\left\|S_{h}\left(0, v_{h}\right)\right\|_{0, \Omega}^{2}+4\left\|\nabla G_{h}\left(0, v_{h}\right)\right\|_{0, \Omega}^{2}+4\left\|\nabla v_{h}\right\|_{0, \Omega}^{2}+2\left\|G_{h}\left(0, v_{h}\right)\right\|_{1, \Omega}^{2} \\
& \leq C\left(\left\|S_{h}\left(0, v_{h}\right)\right\|_{0, \Omega}^{2}+\left\|\nabla v_{h}\right\|_{0, \Omega}^{2}\right) \\
& \leq C\left(\left\|S_{h}\left(0, v_{h}\right)\right\|_{0, \Omega}^{2}+\alpha\left\|\nabla v_{h}\right\|_{0, \Omega}^{2}\right)
\end{aligned}
$$

and

$$
\begin{aligned}
\left\|S_{h}\left(0, v_{h}\right)\right\|_{0, \Omega}^{2}+\alpha\left\|\nabla v_{h}\right\|_{0, \Omega}^{2} & =2\left\|G_{h}\left(0, v_{h}\right)\right\|_{0, \Omega}^{2}+2\left\|v_{h}\right\|_{0, \Omega}^{2}+\alpha\left\|\nabla v_{h}\right\|_{0, \Omega}^{2} \\
& \leq C\left(\left\|v_{h}\right\|_{0, \Omega}^{2}+\alpha\left\|\nabla v_{h}\right\|_{0, \Omega}^{2}\right) \\
& \leq C\left\|v_{h}\right\|_{1, \Omega}^{2}
\end{aligned}
$$

where $S_{h}\left(0, v_{h}\right)=G_{h}\left(0, v_{h}\right)+v_{h}$. Therefore, $\left\|S_{h}\left(0, v_{h}\right)\right\|_{0, \Omega}^{2}+\alpha\left\|\nabla v_{h}\right\|_{0, \Omega}^{2} \approx\left\|v_{h}\right\|_{1, \Omega}^{2}$.

In [6] the authors derived a priori error estimate in the energy norm and $L^{2}$-norm. Here we intend to give a convergence analysis in a simpler way. For compactness we postpone the proof in Appendix A.

Thm:0 Theorem 2.3. Let $(u, y, p) \in H^{1}(\Omega) \times H^{1}(\Omega) \times H_{0}^{1}(\Omega)$ be the solution of the optimal control problem $(2.4)$ and $\left(u_{h}, y_{h}, p_{h}\right) \in V_{h} \times V_{h} \times V_{h}^{0}$ be the solution of the discrete control problems $\sqrt{2.12}$. Assume that $\Omega$ is convex. Then we have

$$
\left\|u-u_{h}\right\|_{1, \Omega}+\left\|y-y_{h}\right\|_{1, \Omega}+\left\|p-p_{h}\right\|_{1, \Omega} \leq C h\left(\|f\|_{0, \Omega}+\left\|y^{d}\right\|_{0, \Omega}\right) .
$$




\section{A POSTERIORI ERROR ESTIMATE}

Now we are in the position to derive a posteriori error estimates. To begin with, we introduce some auxiliary problems: Find $\left(y^{f}\left(u_{h}\right), p\left(y_{h}\right), \hat{u}\right) \in H_{0}^{1}(\Omega) \times H_{0}^{1}(\Omega) \times H^{1}(\Omega)$ such that

aux_6

$$
\left\{\begin{array}{l}
a\left(y^{f}\left(u_{h}\right), w\right)=(f, w)-a\left(u_{h}, w\right) \quad \forall w \in H_{0}^{1}(\Omega) \\
a\left(w, p\left(y_{h}\right)\right)=\left(y_{h}-y^{d}, w\right) \quad \forall w \in H_{0}^{1}(\Omega) ; \\
\alpha a(\hat{u}, v)+(\hat{u}, v)=a\left(v, p_{h}\right)+\left(y^{d}-y_{h}^{f}, v\right) \quad \forall v \in H^{1}(\Omega) .
\end{array}\right.
$$

It is clear that $y_{h}^{f}$ and $p_{h}$ are the finite element approximations of $y^{f}\left(u_{h}\right)$ and $p\left(y_{h}\right)$ in $V_{0}^{h}$, respectively. Moreover, $u_{h}$ is the finite element approximation of $\hat{u}$ in $V_{h}$ in the sense of 2.13 . Furthermore, we define $y^{f}(\hat{u}) \in H_{0}^{1}(\Omega)$ such that

aux_61

$$
a\left(y^{f}(\hat{u}), w\right)=(f, w)-a(\hat{u}, w) \quad \forall w \in H_{0}^{1}(\Omega) .
$$

We set $y(u):=S(f, u)=y^{f}(u)+u$ and $y(\hat{u}):=S(f, \hat{u})=y^{f}(\hat{u})+\hat{u}$.

Thm: 1 Theorem 3.1. Let $(u, y, p) \in H^{1}(\Omega) \times H^{1}(\Omega) \times H_{0}^{1}(\Omega)$ be the solution of the optimal control problem $(2.4)$ and $\left(u_{h}, y_{h}, p_{h}\right) \in V_{h} \times V_{h} \times V_{h}^{0}$ be the solution of the discrete control problems $\left.\sqrt{2.12}\right)$. Let $\left(y^{f}\left(u_{h}\right), p\left(y_{h}\right), \hat{u}\right) \in$ $H_{0}^{1}(\Omega) \times H_{0}^{1}(\Omega) \times H^{1}(\Omega)$ be the solutions of the auxiliary problems $(3.1)$. Then we have

6_est_1

$$
\begin{aligned}
& \left\|u-u_{h}\right\|_{1, \Omega}+\left\|y-y_{h}\right\|_{1, \Omega}+\left\|p-p_{h}\right\|_{1, \Omega} \\
\approx & \left\|\hat{u}-u_{h}\right\|_{1, \Omega}+\left\|y^{f}\left(u_{h}\right)-y_{h}^{f}\right\|_{1, \Omega}+\left\|p\left(y_{h}\right)-p_{h}\right\|_{1, \Omega} .
\end{aligned}
$$

Proof. At first, we prove the upper bound. From (2.4), (3.1) and 3.2 we have

6_est_3

6_est_4

$$
\begin{array}{r}
a\left(y^{f}-y^{f}(\hat{u}), w\right)=a(\hat{u}-u, w) \quad \forall w \in H_{0}^{1}(\Omega) ; \\
a\left(w, p-p\left(y_{h}\right)\right)=\left(y-y_{h}, w\right) \quad \forall w \in H_{0}^{1}(\Omega) ; \\
\alpha a(u-\hat{u}, v)+(u-\hat{u}, v)=a\left(v, p-p_{h}\right)+\left(y_{h}^{f}-y^{f}, v\right) \quad \forall v \in H^{1}(\Omega) .
\end{array}
$$

Setting $w=p-p\left(y_{h}\right)$ in 3.4 and $w=y^{f}-y^{f}(\hat{u})$ in 3.5 we are led to

6_est_5 (3.7)

$$
a\left(\hat{u}-u, p-p\left(y_{h}\right)\right)=\left(y-y_{h}, y^{f}-y^{f}(\hat{u})\right) .
$$

From the triangle inequality it suffices to prove $\|u-\hat{u}\|_{1, \Omega}$. We can derive by setting $v=u-\hat{u}$ in (3.6) that

$$
\begin{aligned}
\alpha\|\nabla(u-\hat{u})\|_{0, \Omega}^{2}= & a\left(u-\hat{u}, p-p_{h}\right)+\left(y_{h}^{f}-y^{f}, u-\hat{u}\right)-(u-\hat{u}, u-\hat{u}) \\
= & a\left(u-\hat{u}, p-p\left(y_{h}\right)\right)+a\left(u-\hat{u}, p\left(y_{h}\right)-p_{h}\right)+\left(y_{h}^{f}-y^{f}, u-\hat{u}\right) \\
& -(u-\hat{u}, u-\hat{u})+a\left(\hat{u}-u, p-p\left(y_{h}\right)\right)+\left(y_{h}-y, y^{f}-y^{f}(\hat{u})\right) \\
= & a\left(u-\hat{u}, p\left(y_{h}\right)-p_{h}\right)+\left(y_{h}^{f}-y^{f}, u-\hat{u}\right) \\
& +(u-\hat{u}, \hat{u}-u)+\left(y_{h}-y, y^{f}-y^{f}(\hat{u})\right) .
\end{aligned}
$$

Note that

$$
\begin{aligned}
& \left(y_{h}^{f}-y^{f}, u-\hat{u}\right)+(u-\hat{u}, \hat{u}-u)+\left(y_{h}-y, y^{f}-y^{f}(\hat{u})\right) \\
= & \left(y_{h}^{f}-y^{f}, u-\hat{u}\right)+(u-\hat{u}, \hat{u}-u)+\left(y_{h}-y, y-y(\hat{u})\right)+\left(y_{h}-y, \hat{u}-u\right) \\
= & \left(u-u_{h}, u-\hat{u}\right)+(u-\hat{u}, \hat{u}-u)+\left(y_{h}-y(\hat{u}), y-y(\hat{u})\right)+(y(\hat{u})-y, y-y(\hat{u})) \\
= & -\|y-y(\hat{u})\|_{0, \Omega}^{2}+\left(u-\hat{u}, \hat{u}-u_{h}\right)+\left(y_{h}-y(\hat{u}), y-y(\hat{u})\right) .
\end{aligned}
$$

Therefore, we are led to

$$
\begin{aligned}
& \alpha\|\nabla(u-\hat{u})\|_{0, \Omega}^{2}+\|y-y(\hat{u})\|_{0, \Omega}^{2} \\
= & a\left(u-\hat{u}, p\left(y_{h}\right)-p_{h}\right)+\left(u-\hat{u}, \hat{u}-u_{h}\right)+\left(y_{h}-y(\hat{u}), y-y(\hat{u})\right) .
\end{aligned}
$$

Moreover, we can derive

$$
\begin{aligned}
\left\|y_{h}-y(\hat{u})\right\|_{0, \Omega} & =\left\|G_{h}\left(f, u_{h}\right)+u_{h}-G(f, \hat{u})-\hat{u}\right\|_{0, \Omega} \\
& \leq C\left(\left\|\hat{u}-u_{h}\right\|_{0, \Omega}+\left\|G_{h}\left(f, u_{h}\right)-G\left(f, u_{h}\right)\right\|_{0, \Omega}+\left\|G\left(f, u_{h}\right)-G(f, \hat{u})\right\|_{0, \Omega}\right) \\
& \leq C\left(\left\|\hat{u}-u_{h}\right\|_{0, \Omega}+\left\|y^{f}\left(u_{h}\right)-y_{h}^{f}\right\|_{1, \Omega}+\left\|\nabla\left(u_{h}-\hat{u}\right)\right\|_{0, \Omega}\right) .
\end{aligned}
$$


It follows from Lemma 2.2 that $\|y-y(\hat{u})\|_{0, \Omega}^{2}+\alpha\|\nabla(u-\hat{u})\|_{0, \Omega}^{2} \approx\|u-\hat{u}\|_{1, \Omega}^{2}$. Cauchy-Schwarz and Young's inequalities yield

$$
\begin{aligned}
& \alpha\|\nabla(u-\hat{u})\|_{0, \Omega}^{2}+\|y-y(\hat{u})\|_{0, \Omega}^{2} \\
\lesssim & \left\|\nabla\left(\hat{u}-u_{h}\right)\right\|_{0, \Omega}^{2}+\left\|p\left(y_{h}\right)-p_{h}\right\|_{1, \Omega}^{2}+\left\|y^{f}\left(u_{h}\right)-y_{h}^{f}\right\|_{1, \Omega}^{2}+\left\|\hat{u}-u_{h}\right\|_{0, \Omega}^{2} .
\end{aligned}
$$

We thus arrive at

$$
\|u-\hat{u}\|_{1, \Omega}^{2} \lesssim\left\|\hat{u}-u_{h}\right\|_{1, \Omega}^{2}+\left\|p\left(y_{h}\right)-p_{h}\right\|_{1, \Omega}^{2}+\left\|y^{f}\left(u_{h}\right)-y_{h}^{f}\right\|_{1, \Omega}^{2} .
$$

Note that $y(\hat{u})-y_{h}=\hat{u}-u_{h}+G(f, \hat{u})-G_{h}\left(f, u_{h}\right)$ and $y-y(\hat{u})=u-\hat{u}+G(f, u)-G(f, \hat{u})$. Therefore, it follows that

$$
\begin{aligned}
\left\|y-y_{h}\right\|_{1, \Omega} & \lesssim\|y-y(\hat{u})\|_{1, \Omega}+\left\|y(\hat{u})-y_{h}\right\|_{1, \Omega} \\
& \lesssim\|u-\hat{u}\|_{1, \Omega}+\|G(f, u)-G(f, \hat{u})\|_{1, \Omega}+\left\|\hat{u}-u_{h}\right\|_{1, \Omega}+\left\|G(f, \hat{u})-G_{h}\left(f, u_{h}\right)\right\|_{1, \Omega} \\
& \lesssim\|u-\hat{u}\|_{1, \Omega}+\left\|\hat{u}-u_{h}\right\|_{1, \Omega}+\left\|G(f, \hat{u})-G\left(f, u_{h}\right)\right\|_{1, \Omega}+\left\|G\left(f, u_{h}\right)-G_{h}\left(f, u_{h}\right)\right\|_{1, \Omega} \\
& \lesssim\|u-\hat{u}\|_{1, \Omega}+\left\|\hat{u}-u_{h}\right\|_{1, \Omega}+\left\|y^{f}\left(u_{h}\right)-y_{h}^{f}\right\|_{1, \Omega}
\end{aligned}
$$

and

$$
\left\|p-p\left(y_{h}\right)\right\|_{1, \Omega} \lesssim\left\|y-y_{h}\right\|_{0, \Omega} .
$$

We thus complete the proof of the upper bound.

Now we turn to the proof of the lower bound. It follows from the triangle inequality that

$$
\begin{aligned}
\left\|p_{h}-p\left(y_{h}\right)\right\|_{1, \Omega} & \lesssim\left\|p_{h}-p\right\|_{1, \Omega}+\left\|p\left(y_{h}\right)-p\right\|_{1, \Omega} \\
& \lesssim\left\|p_{h}-p\right\|_{1, \Omega}+\left\|y-y_{h}\right\|_{1, \Omega}
\end{aligned}
$$

and

$$
\begin{aligned}
\left\|y^{f}\left(u_{h}\right)-y_{h}^{f}\right\|_{1, \Omega} & \lesssim\left\|y^{f}-y_{h}^{f}\right\|_{1, \Omega}+\left\|y^{f}\left(u_{h}\right)-y^{f}\right\|_{1, \Omega} \\
& \lesssim\left\|y-y_{h}\right\|_{1, \Omega}+\left\|u-u_{h}\right\|_{1, \Omega}+\left\|y^{f}\left(u_{h}\right)-y^{f}\right\|_{1, \Omega} \\
& \lesssim\left\|y-y_{h}\right\|_{1, \Omega}+\left\|u-u_{h}\right\|_{1, \Omega} .
\end{aligned}
$$

Moreover, we can conclude from (3.6) that

$$
\begin{aligned}
\alpha\|\nabla(u-\hat{u})\|_{0, \Omega}^{2}+\|u-\hat{u}\|_{0, \Omega}^{2} & \lesssim\left\|\nabla\left(p-p_{h}\right)\right\|_{0, \Omega}^{2}+\left\|y_{h}^{f}-y^{f}\right\|_{0, \Omega}^{2} \\
& \lesssim\left\|\nabla\left(p-p_{h}\right)\right\|_{0, \Omega}^{2}+\left\|u-u_{h}\right\|_{1, \Omega}^{2}+\left\|y-y_{h}\right\|_{1, \Omega}^{2},
\end{aligned}
$$

this together with the triangle inequality yields

$$
\begin{aligned}
\left\|\hat{u}-u_{h}\right\|_{1, \Omega} & \leq\|\hat{u}-u\|_{1, \Omega}+\left\|u-u_{h}\right\|_{1, \Omega} \\
& \lesssim\left\|u-u_{h}\right\|_{1, \Omega}+\left\|p-p_{h}\right\|_{1, \Omega}+\left\|y-y_{h}\right\|_{1, \Omega} .
\end{aligned}
$$

Combining the above estimates we prove the lower bound.

Remark 3.2. In [6. Lemma 5.1] the authors derived a posteriori error estimates for the above Dirichlet boundary control problems in energy space. The authors introduced the following auxiliary problem: Find $\hat{u} \in H^{1}(\Omega)$ such that

$$
\alpha a(\hat{u}, v)=a\left(v, p_{h}\right)+\left(y^{d}-y_{h}, v\right) \quad \forall v \in H^{1}(\Omega) .
$$

However, it is obvious that the above equation does not admit a unique solution since $a(\cdot, \cdot)$ is not coercive in $H^{1}(\Omega)$. Moreover, the fact that $\|\nabla(u-\hat{u})\|_{0, \Omega}$ is not a norm in $H^{1}(\Omega)$ also causes some problems to prove Lemma 5.1 in [6]. In current paper we are able to rigorously derive a posteriori error estimate with the aid of 2.8$)$ and the correct auxiliary problem (3.1). 
To derive a posteriori error estimates for the optimal control problem we introduce some notations. For each element $T \in \mathcal{T}_{h}$, we define the local error indicators $\eta_{u, h}\left(u_{h}, y_{h}, p_{h}, T\right), \eta_{y, h}\left(y_{h}, T\right)$ and $\eta_{p, h}\left(y_{h}, p_{h}, T\right)$ by

local_u

local_y

local_p

estimator_u

estimator_y

estimator_p

$$
\begin{aligned}
\eta_{u, h}\left(u_{h}, y_{h}, p_{h}, T\right):= & \left(h_{T}^{2}\left\|y^{d}-y_{h}\right\|_{0, T}^{2}+\sum_{E \in \mathcal{E}_{h}^{i}, E \subset \partial T} h_{E}\left\|\left[\nabla\left(\alpha u_{h}-p_{h}\right) \cdot n_{E}\right]\right\|_{0, E}^{2}\right. \\
& \left.+\sum_{E \in \mathcal{E}_{h}^{b}, E \subset \partial T} h_{E}\left\|\left[\nabla\left(\alpha u_{h}-p_{h}\right) \cdot n_{E}\right]\right\|_{0, E}^{2}\right)^{\frac{1}{2}}, \\
\eta_{y, h}\left(y_{h}, T\right):= & \left(h_{T}^{2}\|f\|_{0, T}^{2}+\sum_{E \in \mathcal{E}_{h}^{i}, E \subset \partial T} h_{E}\left\|\left[\nabla y_{h} \cdot n_{E}\right]\right\|_{0, E}^{2}\right)^{\frac{1}{2}}, \\
\eta_{p, h}\left(y_{h}, p_{h}, T\right):= & \left(h_{T}^{2}\left\|y_{h}-y^{d}\right\|_{0, T}^{2}+\sum_{E \in \mathcal{E}_{h}^{i}, E \subset \partial T} h_{E}\left\|\left[\nabla p_{h} \cdot n_{E}\right]\right\|_{0, E}^{2}\right)^{\frac{1}{2}},
\end{aligned}
$$

where [ $\left.\nabla v_{h} \cdot n_{E}\right]$ denotes the jump of the outward normal of $v_{h}$ across the edge $E$ with outward normal vector $n_{E}$. Then on a subset $\omega \subset \mathcal{T}_{h}$, we define the error estimators $\eta_{u, h}\left(u_{h}, p_{h}, \omega\right), \eta_{y, h}\left(u_{h}, y_{h}, \omega\right)$ and $\eta_{p, h}\left(y_{h}, p_{h}, \omega\right)$ by

$$
\begin{array}{r}
\eta_{u, h}\left(u_{h}, y_{h}, p_{h}, \omega\right):=\left(\sum_{T \in \mathcal{T}_{h}, T \subset \omega} \eta_{u, h}^{2}\left(u_{h}, y_{h}, p_{h}, T\right)\right)^{\frac{1}{2}}, \\
\eta_{y, h}\left(y_{h}, \omega\right):=\left(\sum_{T \in \mathcal{T}_{h}, T \subset \omega} \eta_{y, h}^{2}\left(y_{h}, T\right)\right)^{\frac{1}{2}}, \\
\eta_{p, h}\left(y_{h}, p_{h}, \omega\right):=\left(\sum_{T \in \mathcal{T}_{h}, T \subset \omega} \eta_{p, h}^{2}\left(y_{h}, p_{h}, T\right)\right)^{\frac{1}{2}} .
\end{array}
$$

Thus, $\eta_{u, h}\left(u_{h}, y_{h}, p_{h}, \mathcal{T}_{h}\right), \eta_{y, h}\left(y_{h}, \mathcal{T}_{h}\right)$ and $\eta_{p, h}\left(y_{h}, p_{h}, \mathcal{T}_{h}\right)$ constitute the error estimators for the control equation, the state equation and the adjoint state equation on $\mathcal{T}_{h}$. For ease of exposition we also define the following quantities:

$$
\begin{aligned}
\eta_{h}^{2}\left(\left(u_{h}, y_{h}, p_{h}\right), T\right) & =\eta_{u, h}^{2}\left(u_{h}, y_{h}, p_{h}, T\right)+\eta_{y, h}^{2}\left(y_{h}, T\right)+\eta_{p, h}^{2}\left(y_{h}, p_{h}, T\right), \\
\operatorname{osc}^{2}\left(\left(u_{h}, y_{h}, p_{h}\right), T\right) & =\operatorname{osc}^{2}(f, T)+\operatorname{osc}^{2}\left(y_{h}-y^{d}, T\right),
\end{aligned}
$$

and the straightforward modifications for $\eta_{h}^{2}\left(\left(u_{h}, y_{h}, p_{h}\right), \mathcal{T}_{h}\right)$ and $\operatorname{osc}^{2}\left(\left(u_{h}, y_{h}, p_{h}\right), \mathcal{T}_{h}\right)$

Now we can derive the following a posteriori upper bound.

Lm:1 Lemma 3.3. Let $\left(u_{h}, y_{h}^{f}, p_{h}\right) \in V_{h} \times V_{h}^{0} \times V_{h}^{0}$ be the solution of the optimal control problem 2.12 and $\left(y^{f}\left(u_{h}\right), p\left(y_{h}\right), \hat{u}\right) \in H_{0}^{1}(\Omega) \times H_{0}^{1}(\Omega) \times H^{1}(\Omega)$ be the solution of the auxiliary problems 3.1$)$. Then we have

$$
\begin{array}{r}
\left\|\hat{u}-u_{h}\right\|_{1, \Omega} \lesssim \eta_{u, h}\left(u_{h}, y_{h}, p_{h}, \mathcal{T}_{h}\right), \\
\left\|y^{f}\left(u_{h}\right)-y_{h}^{f}\right\|_{1, \Omega} \lesssim \eta_{y, h}\left(u_{h}, \mathcal{T}_{h}\right), \\
\left\|p\left(y_{h}\right)-p_{h}\right\|_{1, \Omega} \lesssim \eta_{p, h}\left(y_{h}, p_{h}, \mathcal{T}_{h}\right) .
\end{array}
$$

Proof. From 2.12 and 3.1 we have

$$
\alpha a\left(\hat{u}-u_{h}, v\right)+\left(\hat{u}-u_{h}, v\right)=a\left(v, p_{h}\right)+\left(y^{d}-y_{h}^{f}, v\right)-\alpha a\left(u_{h}, v\right)-\left(u_{h}, v\right) \quad \forall v \in H^{1}(\Omega) .
$$


By setting $v=\hat{u}-u_{h}-\pi_{h}\left(\hat{u}-u_{h}\right)$ where $\pi_{h}: H^{1}(\Omega) \rightarrow V_{h}$ is the Clément-type quasi-interpolation operator (see $[8]$ ), we can derive from the orthogonality and the interpolation error estimates that

$$
\begin{aligned}
c\left\|\hat{u}-u_{h}\right\|_{1, \Omega}^{2}= & \sum_{T \in \mathcal{T}_{h}}\left(\int_{T}\left(y^{d}-y_{h}-\Delta p_{h}+\alpha \Delta u_{h}\right) v d x+\int_{\partial T}\left(\frac{\partial p_{h}}{\partial n}-\alpha \frac{\partial u_{h}}{\partial n}\right) v d s\right) \\
= & \sum_{T \in \mathcal{T}_{h}} \int_{T}\left(y^{d}-y_{h}\right) v d x+\sum_{E \in \mathcal{E}_{h}^{i}} \int_{E}\left[\frac{\partial p_{h}}{\partial n}-\alpha \frac{\partial u_{h}}{\partial n}\right] v d s+\sum_{E \in \mathcal{E}_{h}^{b}} \int_{E}\left(\frac{\partial p_{h}}{\partial n}-\alpha \frac{\partial u_{h}}{\partial n}\right) v d s \\
\lesssim & \left(\sum_{T \in \mathcal{T}_{h}} h_{T}^{2}\left\|y^{d}-y_{h}\right\|_{0, T}^{2}+\sum_{E \in \mathcal{E}_{h}^{i}} h_{E}\left\|\left[\frac{\partial p_{h}}{\partial n}-\alpha \frac{\partial u_{h}}{\partial n}\right]\right\|_{0, E}^{2}\right. \\
& \left.+\sum_{E \in \mathcal{E}_{h}^{b}} h_{E}\left\|\frac{\partial p_{h}}{\partial n}-\alpha \frac{\partial u_{h}}{\partial n}\right\|_{0, E}^{2}\right)^{\frac{1}{2}}\left\|\hat{u}-u_{h}\right\|_{1, \Omega} .
\end{aligned}
$$

We also have

$$
a\left(y^{f}\left(u_{h}\right)-y_{h}^{f}, w\right)=(f, w)-a\left(u_{h}, w\right)-a\left(y_{h}^{f}, w\right) .
$$

By setting $w=\left(y^{f}\left(u_{h}\right)-y_{h}^{f}\right)-\pi_{h}\left(y^{f}\left(u_{h}\right)-y_{h}^{f}\right)$ with $\pi_{h}: H_{0}^{1}(\Omega) \rightarrow V_{h}^{0}$ the Scott-Zhang type interpolation operator (see [32]) we have

$$
\begin{aligned}
\left\|y^{f}\left(u_{h}\right)-y_{h}^{f}\right\|_{1, \Omega}^{2} & =\sum_{T \in \mathcal{T}_{h}}\left(\int_{T}\left(f+\Delta u_{h}+\Delta y_{h}^{f}\right) w d x-\int_{\partial T}\left(\frac{\partial u_{h}}{\partial n}+\frac{\partial y_{h}^{f}}{\partial n}\right) w d s\right) \\
& \lesssim\left(\sum_{T \in \mathcal{T}_{h}} h_{T}^{2}\left\|f+\Delta y_{h}\right\|_{0, T}^{2}+\sum_{E \in \mathcal{E}_{h}^{i}} h_{E}\left\|\left[\frac{\partial y_{h}}{\partial n}\right]\right\|_{0, E}^{2}\right)^{\frac{1}{2}}\left\|y^{f}\left(u_{h}\right)-y_{h}^{f}\right\|_{1, \Omega} .
\end{aligned}
$$

Similarly, we can derive

$$
\left\|p\left(y_{h}\right)-p_{h}\right\|_{1, \Omega}^{2} \lesssim \sum_{T \in \mathcal{T}_{h}} h_{T}^{2}\left\|y_{h}-y^{d}+\Delta p_{h}\right\|_{0, T}^{2}+\sum_{E \in \mathcal{E}_{h}^{i}} h_{E}\left\|\left[\frac{\partial p_{h}}{\partial n}\right]\right\|_{0, E}^{2} .
$$

This completes the proof.

Then we have the following a posteriori lower bound.

Lm:2 Lemma 3.4. Let $\left(u_{h}, y_{h}^{f}, p_{h}\right) \in V_{h} \times V_{h}^{0} \times V_{h}^{0}$ be the solution of the optimal control problem 2.12 and $\left(y^{f}\left(u_{h}\right), p\left(y_{h}\right), \hat{u}\right) \in H_{0}^{1}(\Omega) \times H_{0}^{1}(\Omega) \times H^{1}(\Omega)$ be the solution of the auxiliary problems 3.1$)$. Then we have

$$
\begin{array}{r}
\eta_{u, h}\left(u_{h}, y_{h}, p_{h}, \mathcal{T}_{h}\right) \lesssim\left\|\hat{u}-u_{h}\right\|_{1, \Omega}+\left\|p\left(y_{h}\right)-p_{h}\right\|_{1, \Omega}+\operatorname{osc}\left(y_{h}-y^{d}, \mathcal{T}_{h}\right), \\
\eta_{y, h}\left(y_{h}, \mathcal{T}_{h}\right) \lesssim\left\|\hat{u}-u_{h}\right\|_{1, \Omega}+\left\|y^{f}\left(u_{h}\right)-y_{h}^{f}\right\|_{1, \Omega}+\operatorname{osc}\left(f, \mathcal{T}_{h}\right), \\
\eta_{p, h}\left(y_{h}, p_{h}, \mathcal{T}_{h}\right) \lesssim\left\|p\left(y_{h}\right)-p_{h}\right\|_{1, \Omega}+\operatorname{osc}\left(y_{h}-y^{d}, \mathcal{T}_{h}\right) .
\end{array}
$$

Proof. By using the bubble function techniques of [36] we can prove the lower bound. For simplicity we omit the proof.

Then we can derive reliable and efficient a posteriori error estimators for the finite element approximations of the Dirichlet boundary control problems by collecting the results of Theorem 3.1. Lemmas 3.3 and 3.4

Thm:2 Theorem 3.5. Let $(u, y, p) \in H^{1}(\Omega) \times H^{1}(\Omega) \times H_{0}^{1}(\Omega)$ be the solution of the optimal control problem 2.4 and $\left(u_{h}, y_{h}, p_{h}\right) \in V_{h} \times V_{h} \times V_{h}^{0}$ be the solution of the discrete control problems 2.12). Then we have

$$
\left\|u-u_{h}\right\|_{1, \Omega}+\left\|y-y_{h}\right\|_{1, \Omega}+\left\|p-p_{h}\right\|_{1, \Omega} \leq C_{1} \eta_{h}\left(\left(u_{h}, y_{h}, p_{h}\right), \mathcal{T}_{h}\right)
$$

and

$$
\begin{aligned}
\eta_{h}\left(\left(u_{h}, y_{h}, p_{h}\right), \mathcal{T}_{h}\right) \leq & C_{2}\left(\left\|u-u_{h}\right\|_{1, \Omega}+\left\|y-y_{h}\right\|_{1, \Omega}+\left\|p-p_{h}\right\|_{1, \Omega}\right) \\
& +C_{3} \operatorname{Osc}\left(\left(u_{h}, y_{h}, p_{h}\right), \mathcal{T}_{h}\right) .
\end{aligned}
$$




\section{AdAPTIVE ALGORITHM FOR THE OPTIMAL CONTROL PROBLEMS AND ITS CONVERGENCE}

In this section we present the adaptive finite element algorithm to solve the Dirichlet boundary control problems. By establishing some properties for the energy norm errors of the control, the state and adjoint state we prove the convergence of the adaptive algorithm.

4.1. Adaptive algorithm. The adaptive finite element procedure consists of the following loops

$$
\mathrm{SOLVE} \rightarrow \text { ESTIMATE } \rightarrow \text { MARK } \rightarrow \text { REFINE }
$$

The ESTIMATE step is based on the a posteriori error indicators presented in Section 3, while the step REFINE can be done by using iterative or recursive bisection of elements with the minimal refinement condition (see [36]). There are several alternatives for MARK procedure like Max strategy or Dörfler's strategy ([10]). Note that there are three error estimators $\eta_{u, h}\left(u_{h}, y_{h}, p_{h}, T\right), \eta_{y, h}\left(y_{h}, T\right)$ and $\eta_{p, h}\left(y_{h}, p_{h}, T\right)$ contributed to the control approximation, the state approximation and adjoint state approximation, respectively. We use the sum of them as our error indicators.

To begin with, let $\mathcal{T}_{0}$ be a conforming and quasi-uniform partition of $\bar{\Omega}$ into disjoint triangles. Each element in $\mathcal{T}_{0}$ is assumed to be shape regular in the usual sense (see [7]). We denote the set of all conforming descendants $\mathcal{T}$ of $\mathcal{T}_{0}$ by $\mathbb{T}$, which can be generated through uniform or local refinements by newest vertex bisection algorithm. Given a fixed number $b \geq 1$, for any $\mathcal{T}_{h_{k}} \in \mathbb{T}$ and $\mathcal{M}_{h_{k}} \subset \mathcal{T}_{h_{k}}$ of marked elements,

$$
\mathcal{T}_{h_{k+1}}=\operatorname{REFINE}\left(\mathcal{T}_{h_{k}}, \mathcal{M}_{h_{k}}\right)
$$

outputs a conforming triangulation $\mathcal{T}_{h_{k+1}} \in \mathbb{T}$, where at least all elements of $\mathcal{M}_{h_{k}}$ are bisected $b$ times. We denote $\omega_{T}$ the patch of elements sharing a vertex or a facet with $T$.

In the following, we frequently use the notations $V_{k}$ and $\mathcal{T}_{k}$ to denote $V_{h_{k}}$ and $\mathcal{T}_{h_{k}}$. We also denote $\left(u_{h_{k}}, y_{h_{k}}, p_{h_{k}}\right)$ by $\left(u_{k}, y_{k}, p_{k}\right)$. Now we describe the adaptive finite element algorithm for the optimal control problem (2.12) as follows:

Alg:3.1 Algorithm 4.1. Adaptive finite element algorithm for OCPs:

(1) Given an initial mesh $\mathcal{T}_{0}$ with mesh size $h_{0}$ and the associated finite element spaces $V_{0}$ and $V_{0}^{0}$.

(2) Set $k=0$ and solve the optimal control problem $\sqrt{2.12}$ to obtain $\left(u_{k}, y_{k}, p_{k}\right) \in V_{k} \times V_{k} \times V_{k}^{0}$.

(3) Compute the local error indicator $\eta_{k}\left(\left(u_{k}, y_{k}, p_{k}\right), T\right)$.

(4) Construct $\mathcal{M}_{k} \subset \mathcal{T}_{k}$ by some appropriate marking algorithms.

(5) Refine $\mathcal{M}_{k}$ to get a new conforming mesh $\mathcal{T}_{k+1}$ by procedure REFINE using bisection algorithm.

(6) Construct the finite element spaces $V_{k+1}$ and $V_{k+1}^{0}$, solve the optimal control problem (2.12) to $\operatorname{obtain}\left(u_{k+1}, y_{k+1}, p_{k+1}\right) \in V_{k+1} \times V_{k+1} \times V_{k+1}^{0}$.

(7) Set $k=k+1$ and go to Step (3).

For the marking algorithm we require the following property holds

$$
\max _{T \in \mathcal{T}_{k}} \eta_{k}\left(\left(u_{k}, y_{k}, p_{k}\right), T\right) \leq \max _{T \in \mathcal{M}_{k}} \eta_{k}\left(\left(u_{k}, y_{k}, p_{k}\right), T\right)
$$

This property allows many marking algorithms, for example, the well-known bulk criteria selects a minimal subset $\mathcal{M}_{k} \subset \mathcal{T}_{k}$ such that

$$
\sum_{T \in \mathcal{M}_{k}} \eta_{k}^{2}\left(\left(u_{k}, y_{k}, p_{k}\right), T\right) \geq \theta \eta_{k}^{2}\left(\left(u_{k}, y_{k}, p_{k}\right), \mathcal{T}_{k}\right)
$$

and the Max strategy chooses elements satisfying

$$
\forall T \in \mathcal{M}_{k}: \quad \eta_{k}\left(\left(u_{k}, y_{k}, p_{k}\right), T\right) \geq \theta \max _{T \in \mathcal{T}_{k}} \eta_{k}\left(\left(u_{k}, y_{k}, p_{k}\right), T\right),
$$

where $\theta \in(0,1)$ is referred to marking parameter. 
4.2. Convergence to the limiting problem. In this subsection we prove the convergence of the sequence $\left\{\left(u_{k}, y_{k}, p_{k}\right)\right\}$ generated by Algorithm 4.1 to the solution of a limit optimal control problem. To begin with, we define two limiting spaces

$$
V_{\infty}:=\overline{\bigcup_{k \geq 0} V_{k}}\left(\text { in } H^{1}(\Omega)-\text { norm }\right) \quad \text { and } \quad V_{\infty}^{0}:=\overline{\bigcup_{k \geq 0} V_{k}^{0}}\left(\text { in } H_{0}^{1}(\Omega)-\text { norm }\right),
$$

which are well-defined due to the space nesting $V_{k} \subset V_{k+1}$. It is clear that $V_{\infty}$ and $V_{\infty}^{0}$ are closed subspaces of $H^{1}(\Omega)$ and $H_{0}^{1}(\Omega)$, respectively. Then we are able to define a limiting control problem

OCP_limit

OCP_limit_state

tate_weak_limit

OCP_OPT_limit

OCP_OPT_limit

trol_cont_limit

Lm: 3.1

$$
\min _{u \in V_{\infty}} J(y, u)=\frac{1}{2}\left\|y-y^{d}\right\|_{0, \Omega}^{2}+\frac{\alpha}{2}\|\nabla u\|_{0, \Omega}^{2}
$$

subject to

$$
y \in V_{\infty},\left.y\right|_{\Gamma}=u: \quad a(y, v)=(f, v) \quad \forall v \in V_{\infty}^{0} .
$$

Similar to the control problem (1.1)-(1.2) we can prove the above limiting control problem admits a unique solution $\left(u_{\infty}, y_{\infty}\right) \in V_{\infty} \times V_{\infty}$. Let $y_{\infty}=y_{\infty}^{f}+u_{\infty}$ such that $y_{\infty}^{f} \in V_{\infty}^{0}$ and

$$
a\left(y_{\infty}^{f}, w\right)=(f, w)-a\left(u_{\infty}, w\right) \quad \forall w \in V_{\infty}^{0} .
$$

We may introduce the solution operator $G_{\infty}: L^{2}(\Omega) \times H^{1}(\Omega) \rightarrow V_{\infty}^{0}$ associated with 4.4 such that $y_{\infty}^{f}=$ $G_{\infty}\left(f, u_{\infty}\right)$. Therefore, we can introduce the solution operator for the state equation 4.3 as $S_{\infty}: L^{2}(\Omega) \times$ $H^{1}(\Omega) \rightarrow V_{\infty}$ with $y_{\infty}:=S_{\infty}\left(f, u_{\infty}\right)=G_{\infty}\left(f, u_{\infty}\right)+u_{\infty}$.

Now we can derive the first order optimality system of problem $\sqrt{4.2}$ - $-(4.3)$ : There exists $\left(u_{\infty}, y_{\infty}^{f}, p_{\infty}\right) \in$ $V_{\infty} \times V_{\infty}^{0} \times V_{\infty}^{0}$ such that

$$
\left\{\begin{array}{l}
a\left(y_{\infty}^{f}, w\right)=(f, w)-a\left(u_{\infty}, w\right) \quad \forall w \in V_{\infty}^{0} \\
a\left(w, p_{\infty}\right)=\left(y_{\infty}-y^{d}, w\right) \quad \forall w \in V_{\infty}^{0} ; \\
\alpha a\left(u_{\infty}, v\right)=a\left(v, p_{\infty}\right)+\left(y^{d}-y_{\infty}, v\right) \quad \forall v \in V_{\infty}
\end{array}\right.
$$

where $y_{\infty}=y_{\infty}^{f}+u_{\infty} \in V_{\infty}$. As the state equation is self-adjoint we use the notation $p_{\infty}=G_{\infty}\left(y_{\infty}-y^{d}, 0\right)$. From 4.5 we conclude that $u$ is harmonic in the sense that

$$
a\left(u_{\infty}, v\right)=0 \quad \forall v \in V_{\infty}^{0} .
$$

Therefore, $u_{\infty}=S_{\infty}\left(0, u_{\infty}\right)$ and the first equation in 4.5$)$ can be written as

$$
a\left(y_{\infty}^{f}, w\right)=(f, w) \quad \forall w \in V_{\infty}^{0} .
$$

Moreover, the third equation in 4.5 can be written as

$$
\alpha a\left(u_{\infty}, v\right)+\left(u_{\infty}, v\right)=a\left(v, p_{\infty}\right)+\left(y^{d}-y_{\infty}^{f}, v\right) \quad \forall v \in V_{\infty} .
$$

Firstly, we recall the following results concerning the convergence of solution operators $G_{\infty}$ and $S_{\infty}$, whose proof is very similar to that of [30, Lemma 4.2].

Lemma 4.2. For any $f \in L^{2}(\Omega), y-y^{d} \in L^{2}(\Omega)$ and $u \in H^{1}(\Omega)$ we have $G_{k}(f, u) \rightarrow G_{\infty}(f, u), G_{k}(y-$ $\left.y^{d}, 0\right) \rightarrow G_{\infty}\left(y-y^{d}, 0\right)$ and $S_{k}(f, u) \rightarrow S_{\infty}(f, u)$ in $H^{1}(\Omega)$ as $k \rightarrow \infty$.

Secondly, we prove the convergence of the discrete solutions $\left(u_{k}, y_{k}, p_{k}\right)$ to the solutions of limiting control problem 4.2)- 4.3 .

Lm:3.2 Lemma 4.3. Assume that $\left(u_{k}, y_{k}, p_{k}\right) \in V_{k} \times V_{k} \times V_{k}^{0}$ is the solution sequence generated by adaptive Algorithm 4.1. Then we have the strong convergence result

$$
\left\|u_{k}-u_{\infty}\right\|_{1, \Omega}+\left\|y_{k}-y_{\infty}\right\|_{1, \Omega}+\left\|p_{k}-p_{\infty}\right\|_{1, \Omega} \rightarrow 0 \text { as } k \rightarrow \infty .
$$

Proof. The proof is very similar to the proof of Theorem 2.3 First, we introduce some auxiliary problems: Find $\left(y_{k}^{f}\left(u_{\infty}\right), p_{k}\left(y_{\infty}\right), \tilde{u}_{k}\right) \in V_{k}^{0} \times V_{k}^{0} \times V_{k}$ such that

$$
\left\{\begin{array}{l}
a\left(y_{k}^{f}\left(u_{\infty}\right), w_{k}\right)=\left(f, w_{k}\right)-a\left(u_{\infty}, w_{k}\right) \quad \forall w_{k} \in V_{k}^{0} ; \\
a\left(w_{k}, p_{k}\left(y_{\infty}\right)\right)=\left(y_{\infty}-y^{d}, w_{k}\right) \quad \forall w_{k} \in V_{k}^{0} ; \\
\alpha a\left(\tilde{u}_{k}, v_{k}\right)+\left(\tilde{u}_{k}, v_{k}\right)=a\left(v_{k}, p_{\infty}\right)+\left(y^{d}-y_{\infty}^{f}, v_{k}\right) \quad \forall v_{k} \in V_{k} .
\end{array}\right.
$$


It is clear that $y_{k}^{f}\left(u_{\infty}\right)=G_{k}\left(f, u_{\infty}\right)$ and $p_{k}\left(y_{\infty}\right)=G_{k}\left(y_{\infty}-y^{d}, 0\right)$. Moreover, we define $y_{k}^{f}\left(\tilde{u}_{k}\right) \in V_{k}^{0}$ such that

$$
a\left(y_{k}^{f}\left(\tilde{u}_{k}\right), w_{k}\right)=\left(f, w_{k}\right)-a\left(\tilde{u}_{k}, w_{k}\right) \quad \forall w_{k} \in V_{k}^{0} .
$$

We set $y_{k}\left(u_{\infty}\right):=S_{k}\left(f, u_{\infty}\right)=y_{k}^{f}\left(u_{\infty}\right)+u_{\infty}$ and $y_{k}\left(\tilde{u}_{k}\right):=S_{k}\left(f, \tilde{u}_{k}\right)=y_{k}^{f}\left(\tilde{u}_{k}\right)+\tilde{u}_{k}$. It is clear that $y_{k}^{f}\left(u_{\infty}\right)$ and $p_{k}\left(y_{\infty}\right)$ are the finite element approximations of $y_{\infty}^{f}$ and $p_{\infty}$ in $V_{k}^{0}$, respectively. Moreover, $\tilde{u}_{k}$ is the finite element approximation of $u_{\infty}$ in $V_{k}$ in the sense of [4.7]. Lemma 4.2] and [30, Lemma 4.2] imply that

$$
\lim _{k \rightarrow \infty}\left\|\tilde{u}_{k}-u_{\infty}\right\|_{1, \Omega}=0, \quad \lim _{k \rightarrow \infty}\left\|y_{k}^{f}\left(u_{\infty}\right)-y_{\infty}^{f}\right\|_{1, \Omega}=0, \quad \lim _{k \rightarrow \infty}\left\|p_{k}\left(y_{\infty}\right)-p_{\infty}\right\|_{1, \Omega}=0 .
$$

Note that $y_{k}\left(\tilde{u}_{k}\right)-y_{k}=\tilde{u}_{k}-u_{k}+G_{k}\left(f, \tilde{u}_{k}\right)-G_{k}\left(f, u_{k}\right)$ and $y_{\infty}-y_{k}\left(\tilde{u}_{k}\right)=u_{\infty}-\tilde{u}_{k}+G_{\infty}\left(f, u_{\infty}\right)-G_{k}\left(f, \tilde{u}_{k}\right)$. It is not difficult to prove

$$
\begin{aligned}
\left\|y_{\infty}-y_{k}\right\|_{1, \Omega} \leq & \left\|y_{\infty}-y_{k}\left(\tilde{u}_{k}\right)\right\|_{1, \Omega}+\left\|y_{k}\left(\tilde{u}_{k}\right)-y_{k}\right\|_{1, \Omega} \\
\leq & \left\|u_{\infty}-\tilde{u}_{k}\right\|_{1, \Omega}+\left\|G_{\infty}\left(f, u_{\infty}\right)-G_{k}\left(f, \tilde{u}_{k}\right)\right\|_{1, \Omega}+\left\|\tilde{u}_{k}-u_{k}\right\|_{1, \Omega} \\
& +\left\|G_{k}\left(f, \tilde{u}_{k}\right)-G_{k}\left(f, u_{k}\right)\right\|_{1, \Omega} \\
\lesssim & \left\|u_{\infty}-\tilde{u}_{k}\right\|_{1, \Omega}+\left\|\tilde{u}_{k}-u_{k}\right\|_{1, \Omega}+\left\|G_{\infty}\left(f, u_{\infty}\right)-G_{k}\left(f, u_{\infty}\right)\right\|_{1, \Omega} \\
& +\left\|G_{k}\left(f, u_{\infty}\right)-G_{k}\left(f, \tilde{u}_{k}\right)\right\|_{1, \Omega} \\
\lesssim & \left\|u_{\infty}-\tilde{u}_{k}\right\|_{1, \Omega}+\left\|\tilde{u}_{k}-u_{k}\right\|_{1, \Omega}+\left\|y_{\infty}^{f}-y_{k}^{f}\left(u_{\infty}\right)\right\|_{1, \Omega}
\end{aligned}
$$

and

$$
\begin{aligned}
\left\|p_{\infty}-p_{k}\right\|_{1, \Omega} & \leq\left\|p_{\infty}-p_{k}\left(y_{\infty}\right)\right\|_{1, \Omega}+\left\|p_{k}\left(y_{\infty}\right)-p_{k}\right\|_{1, \Omega} \\
& \lesssim\left\|p_{\infty}-p_{k}\left(y_{\infty}\right)\right\|_{1, \Omega}+\left\|y_{\infty}-y_{k}\right\|_{0, \Omega} .
\end{aligned}
$$

From the triangle inequality we also have

$$
\left\|u_{\infty}-u_{k}\right\|_{1, \Omega} \leq\left\|u_{\infty}-\tilde{u}_{k}\right\|_{1, \Omega}+\left\|\tilde{u}_{k}-u_{k}\right\|_{1, \Omega} .
$$

So it suffices to estimate $\left\|\tilde{u}_{k}-u_{k}\right\|_{1, \Omega}$. From (2.12), (4.9) and (4.10) we have

2_est_4

2_est_5

2_est_6

$$
\begin{array}{r}
a\left(y_{k}^{f}-y_{k}^{f}\left(\tilde{u}_{k}\right), w_{k}\right)=a\left(\tilde{u}_{k}-u_{k}, w_{k}\right) \quad \forall w_{k} \in V_{k}^{0} ; \\
a\left(w_{k}, p_{k}\left(y_{\infty}\right)-p_{k}\right)=\left(y_{\infty}-y_{k}, w_{k}\right) \quad \forall w_{k} \in V_{k}^{0} ; \\
\alpha a\left(u_{k}-\tilde{u}_{k}, v_{k}\right)+\left(u_{k}-\tilde{u}_{k}, v_{k}\right)=a\left(v_{k}, p_{k}-p_{\infty}\right)+\left(y_{\infty}^{f}-y_{k}^{f}, v_{k}\right) \quad \forall v_{k} \in V_{k} .
\end{array}
$$

Setting $w_{k}=p_{k}\left(y_{\infty}\right)-p_{k}$ in 4.15 and $w_{k}=y_{k}^{f}-y_{k}^{f}\left(\tilde{u}_{k}\right)$ in 4.16 we are led to

$$
a\left(\tilde{u}_{k}-u_{k}, p_{k}\left(y_{\infty}\right)-p_{k}\right)=\left(y_{\infty}-y_{k}, y_{k}^{f}-y_{k}^{f}\left(\tilde{u}_{k}\right)\right) .
$$

We can derive by setting $v=u_{k}-\tilde{u}_{k}$ in (4.17) that

$$
\begin{aligned}
\alpha\left\|\nabla\left(u_{k}-\tilde{u}_{k}\right)\right\|_{0, \Omega}^{2}= & a\left(u_{k}-\tilde{u}_{k}, p_{k}-p_{\infty}\right)+\left(y_{\infty}^{f}-y_{k}^{f}, u_{k}-\tilde{u}_{k}\right)-\left(u_{k}-\tilde{u}_{k}, u_{k}-\tilde{u}_{k}\right) \\
= & a\left(u_{k}-\tilde{u}_{k}, p_{k}-p_{k}\left(y_{\infty}\right)\right)+a\left(u_{k}-\tilde{u}_{k}, p_{k}\left(y_{\infty}\right)-p_{\infty}\right)+\left(y_{\infty}^{f}-y_{k}^{f}, u_{k}-\tilde{u}_{k}\right) \\
& -\left(u_{k}-\tilde{u}_{k}, u_{k}-\tilde{u}_{k}\right)+a\left(u_{k}-\tilde{u}_{k}, p_{k}\left(y_{\infty}\right)-p_{k}\right)+\left(y_{\infty}-y_{k}, y_{k}^{f}-y_{k}^{f}\left(\tilde{u}_{k}\right)\right) \\
= & a\left(u_{k}-\tilde{u}_{k}, p_{k}\left(y_{\infty}\right)-p_{\infty}\right)+\left(y_{\infty}^{f}-y_{k}^{f}, u_{k}-\tilde{u}_{k}\right) \\
& +\left(u_{k}-\tilde{u}_{k}, \tilde{u}_{k}-u_{k}\right)+\left(y_{\infty}-y_{k}, y_{k}^{f}-y_{k}^{f}\left(\tilde{u}_{k}\right)\right) .
\end{aligned}
$$

Note that

$$
\begin{aligned}
& \left(y_{\infty}^{f}-y_{k}^{f}, u_{k}-\tilde{u}_{k}\right)+\left(u_{k}-\tilde{u}_{k}, \tilde{u}_{k}-u_{k}\right)+\left(y_{\infty}-y_{k}, y_{k}^{f}-y_{k}^{f}\left(\tilde{u}_{k}\right)\right) \\
= & \left(y_{\infty}^{f}-y_{k}^{f}, u_{k}-\tilde{u}_{k}\right)+\left(u_{k}-\tilde{u}_{k}, \tilde{u}_{k}-u_{k}\right)+\left(y_{\infty}-y_{k}, y_{k}-y_{k}\left(\tilde{u}_{k}\right)\right)+\left(y_{\infty}-y_{k}, \tilde{u}_{k}-u_{k}\right) \\
= & \left(u_{k}-u_{\infty}, u_{k}-\tilde{u}_{k}\right)+\left(u_{k}-\tilde{u}_{k}, \tilde{u}_{k}-u_{k}\right)+\left(y_{\infty}-y_{k}\left(\tilde{u}_{k}\right), y_{k}-y_{k}\left(\tilde{u}_{k}\right)\right)+\left(y_{k}\left(\tilde{u}_{k}\right)-y_{k}, y_{k}-y_{k}\left(\tilde{u}_{k}\right)\right) \\
= & -\left\|y_{k}-y_{k}\left(\tilde{u}_{k}\right)\right\|_{0, \Omega}^{2}+\left(\tilde{u}_{k}-u_{\infty}, u_{k}-\tilde{u}_{k}\right)+\left(y_{\infty}-y_{k}\left(\tilde{u}_{k}\right), y_{k}-y_{k}\left(\tilde{u}_{k}\right)\right) .
\end{aligned}
$$


Therefore, we are led to

$$
\begin{aligned}
& \alpha\left\|\nabla\left(u_{k}-\tilde{u}_{k}\right)\right\|_{0, \Omega}^{2}+\left\|y_{k}-y_{k}\left(\tilde{u}_{k}\right)\right\|_{0, \Omega}^{2} \\
= & a\left(u_{k}-\tilde{u}_{k}, p_{k}\left(y_{\infty}\right)-p_{\infty}\right)+\left(\tilde{u}_{k}-u_{\infty}, u_{k}-\tilde{u}_{k}\right)+\left(y_{\infty}-y_{k}\left(\tilde{u}_{k}\right), y_{k}-y_{k}\left(\tilde{u}_{k}\right)\right) .
\end{aligned}
$$

Furthermore, we can derive

$$
\begin{aligned}
\left\|y_{\infty}-y_{k}\left(\tilde{u}_{k}\right)\right\|_{0, \Omega} & =\left\|G_{\infty}\left(f, u_{\infty}\right)+u_{\infty}-G_{k}\left(f, \tilde{u}_{k}\right)-\tilde{u}_{k}\right\|_{0, \Omega} \\
& \leq\left\|\tilde{u}_{k}-u_{\infty}\right\|_{0, \Omega}+\left\|G_{\infty}\left(f, u_{\infty}\right)-G_{k}\left(f, u_{\infty}\right)\right\|_{0, \Omega}+\left\|G_{k}\left(f, u_{\infty}\right)-G_{k}\left(f, \tilde{u}_{k}\right)\right\|_{0, \Omega} \\
& \leq C\left(\left\|\tilde{u}_{k}-u_{\infty}\right\|_{0, \Omega}+\left\|y_{\infty}^{f}-y_{k}^{f}\left(u_{\infty}\right)\right\|_{1, \Omega}+\left\|\nabla\left(\tilde{u}_{k}-u_{\infty}\right)\right\|_{0, \Omega}\right) .
\end{aligned}
$$

We can conclude from Lemma 3.3 that $\left\|y_{k}-y_{k}\left(\tilde{u}_{k}\right)\right\|_{0, \Omega}^{2}+\alpha\left\|\nabla\left(u_{k}-\tilde{u}_{k}\right)\right\|_{0, \Omega}^{2} \approx\left\|u_{k}-\tilde{u}_{k}\right\|_{1, \Omega}^{2}$. Therefore, Cauchy-Schwarz and Young's inequalities give

$$
\begin{aligned}
& \alpha\left\|\nabla\left(u_{k}-\tilde{u}_{k}\right)\right\|_{0, \Omega}^{2}+\left\|y_{k}-y_{k}\left(\tilde{u}_{k}\right)\right\|_{0, \Omega}^{2} \\
\lesssim & \left\|\nabla\left(u_{\infty}-\tilde{u}_{k}\right)\right\|_{0, \Omega}^{2}+\left\|p_{\infty}-p_{k}\left(y_{\infty}\right)\right\|_{1, \Omega}^{2}+\left\|y_{\infty}^{f}-y_{k}^{f}\left(u_{\infty}\right)\right\|_{1, \Omega}^{2}+\left\|u_{\infty}-\tilde{u}_{k}\right\|_{0, \Omega}^{2} .
\end{aligned}
$$

Thus, we arrive at

2_est_8

$$
\left\|u_{k}-\tilde{u}_{k}\right\|_{1, \Omega}^{2} \lesssim\left\|u_{\infty}-\tilde{u}_{k}\right\|_{1, \Omega}^{2}+\left\|p_{\infty}-p_{k}\left(y_{\infty}\right)\right\|_{1, \Omega}^{2}+\left\|y_{\infty}^{f}-y_{k}^{f}\left(u_{\infty}\right)\right\|_{1, \Omega}^{2} .
$$

Combining (4.11)-(4.14) and 4.22) we finish the proof of the theorem.

4.3. Convergence of the error and estimator. In this subsection we intend to prove that the discrete solutions $\left(u_{k}, y_{k}, p_{k}\right)$ generated by Algorithm 4.1 converge to the solutions of continuous optimal control problem $(1.1)-(1.2)$ and the error estimator $\eta_{k}\left(\left(u_{k}, y_{k}, p_{k}\right), \mathcal{T}_{k}\right)$ converges to zero.

Firstly, we introduce a classification of the elements generated by the adaptive algorithm. For each triangulation $\mathcal{T}_{k}$ we define following the line of [33]:

$$
\mathcal{T}_{k}^{+}:=\bigcap_{l \geq k} \mathcal{T}_{l}=\left\{T \in \mathcal{T}_{k}: T \in \mathcal{T}_{l} \quad \forall l \geq k\right\} \quad \text { and } \quad \mathcal{T}_{k}^{0}:=\mathcal{T}_{k} \backslash \mathcal{T}_{k}^{+} .
$$

It is clear that the set $\mathcal{T}_{k}^{+}$consists of all elements that are not refined after $k$-th iteration and the nesting property $\mathcal{T}_{l}^{+} \subset \mathcal{T}_{k}^{+}(k \geq l)$ holds for the sequence $\left\{\mathcal{T}_{k}^{+}\right\}$. On the contrary, the set $\mathcal{T}_{k}^{0}$ contains all elements that are refined at least one time after iteration $k$, i.e., for any $T \in \mathcal{T}_{k}^{0}$, there exists $l \geq k$ such that $T \in \mathcal{T}_{l}$ and $T \notin \mathcal{T}_{l+1}$. We split the domain $\Omega$ into two parts as follows: $\bar{\Omega}=\Omega_{k}^{+} \cup \Omega_{k}^{0}:=\Omega\left(\mathcal{T}_{k}^{+}\right) \cup \Omega\left(\mathcal{T}_{k}^{0}\right)$. We can define the piecewise constant mesh-size function $h_{k}: \bar{\Omega} \rightarrow \mathbb{R}^{+}$so that $\left.h_{k}\right|_{T}:=|T|^{\frac{1}{2}}$. The following convergence of the mesh-size function $h_{k}$ is presented in [30, Lemma 4.3] (see also [33, Corollary 3.3]).

Lm:3.3 Lemma 4.4. Let $\chi_{k}^{0}$ be the characteristic function of $\Omega_{k}^{0}$. Then the mesh-size function $h_{k}$ convergence to zero in $\Omega_{k}^{0}$ in the sense that

$$
\lim _{k \rightarrow \infty}\left\|h_{k} \chi_{k}^{0}\right\|_{L^{\infty}(\Omega)}=\lim _{k \rightarrow \infty}\left\|h_{k}\right\|_{L^{\infty}\left(\Omega_{k}^{0}\right)}=0 .
$$

With the help of the convergence of the mesh-size function $h_{k}$ in $\Omega_{k}^{0}$ we can prove the convergence of the maximal error indicator in $\mathcal{T}_{k}$.

Lm:3.4 Lemma 4.5. Let $\eta_{k}\left(\left(u_{k}, y_{k}, p_{k}\right), T\right), T \in \mathcal{T}_{k}$ be the local error indicator defined in Section 3. Then the following convergence holds:

$$
\lim _{k \rightarrow \infty} \max _{T \in \mathcal{T}_{k}} \eta_{k}\left(\left(u_{k}, y_{k}, p_{k}\right), T\right)=0 .
$$

Proof. Recall the assumption on the marking algorithm in 4.1.

$$
\max _{T \in \mathcal{T}_{k}} \eta_{k}\left(\left(u_{k}, y_{k}, p_{k}\right), T\right) \leq \max _{T \in \mathcal{M}_{k}} \eta_{k}\left(\left(u_{k}, y_{k}, p_{k}\right), T\right),
$$

where $\mathcal{M}_{k}$ is the set of marked elements generated in Algorithm 4.1. Therefore, it suffices to prove

$$
\max _{T \in \mathcal{M}_{k}} \eta_{k}\left(\left(u_{k}, y_{k}, p_{k}\right), T\right) \rightarrow 0 \quad \text { as } k \rightarrow \infty .
$$


Let $T_{k}$ be the element with maximal error indicator among $\mathcal{M}_{k}$. It is clear that $T_{k} \in \mathcal{M}_{k} \subset \mathcal{T}_{k}^{0}$. Using the trace theorem, the inverse inequality and the triangle inequality we can derive

$$
\begin{aligned}
\eta_{u, k}\left(u_{k}, y_{k}, p_{k}, T_{k}\right) \leq & C\left(h_{T_{k}}\left\|y^{d}-y_{k}\right\|_{0, T_{k}}+\left\|\nabla u_{k}\right\|_{0, \omega_{T_{k}}}+\left\|\nabla p_{k}\right\|_{0, \omega_{T_{k}}}\right) \\
\leq & C\left(h_{T_{k}}\left\|y^{d}-y_{\infty}\right\|_{0, T_{k}}+h_{T_{k}}\left\|y_{k}-y_{\infty}\right\|_{1, \Omega}+\left\|\nabla u_{\infty}\right\|_{0, \omega_{T_{k}}}\right. \\
& \left.+\left\|u_{k}-u_{\infty}\right\|_{1, \Omega}+\left\|\nabla p_{\infty}\right\|_{0, \omega_{T_{k}}}+\left\|p_{k}-p_{\infty}\right\|_{1, \Omega}\right), \\
\eta_{y, k}\left(u_{k}, T_{k}\right) \leq & C\left(h_{T_{k}}\|f\|_{0, T_{k}}+\left\|\nabla y_{k}\right\|_{0, \omega_{T_{k}}}\right) \\
\leq & C\left(h_{T_{k}}\|f\|_{0, T_{k}}+\left\|\nabla y_{\infty}\right\|_{0, \omega_{T_{k}}}+\left\|y_{k}-y_{\infty}\right\|_{1, \Omega}\right)
\end{aligned}
$$

and

$$
\begin{aligned}
\eta_{p, k}\left(y_{k}, p_{k}, T_{k}\right) & \leq C\left(h_{T_{k}}\left\|y^{d}-y_{k}\right\|_{0, T_{k}}+\left\|\nabla p_{k}\right\|_{0, \omega_{T_{k}}}\right) \\
& \leq C\left(h_{T_{k}}\left\|y^{d}-y_{\infty}\right\|_{0, T_{k}}+h_{T_{k}}\left\|y_{k}-y_{\infty}\right\|_{1, \Omega}+\left\|\nabla p_{\infty}\right\|_{0, \omega_{T_{k}}}+\left\|p_{k}-p_{\infty}\right\|_{1, \Omega}\right) .
\end{aligned}
$$

It follows from the local quasi-uniformity of $\mathcal{T}_{k}$ and Lemma 4.4 that

$$
\left|\omega_{T_{k}}\right| \leq C\left|T_{k}\right| \leq C\left\|h_{T_{k}}^{2}\right\|_{L^{\infty}\left(\Omega_{k}^{0}\right)} \rightarrow 0 \quad \text { as } k \rightarrow \infty .
$$

Thus, the terms involving the integrals on $T_{k}$ or $\omega_{T_{k}}$ vanish as $k \rightarrow \infty$ by the continuity of $\|\cdot\|_{0, \Omega}$ with respect to the Lebesgue measure. The terms involving the difference of $\left(u_{k}, y_{k}, p_{k}\right)$ and $\left(u_{\infty}, y_{\infty}, p_{\infty}\right)$ converge due to Lemma 4.3 . We thus prove that $\eta_{k}\left(\left(u_{k}, y_{k}, p_{k}\right), T_{k}\right) \rightarrow 0$ as $k \rightarrow \infty$. The assertion of the lemma follows immediately.

For the following purpose we introduce the residuals with respect to the control equation, the state equation and the adjoint state equation:

$$
\begin{aligned}
\left\langle\mathcal{R}_{u}\left(u_{k}, y_{k}, p_{k}\right), v\right\rangle=a\left(v, p_{k}\right)+\left(y^{d}-y_{k}, v\right)-\alpha a\left(u_{k}, v\right) & \forall v \in H^{1}(\Omega), \\
\left\langle\mathcal{R}_{y}\left(u_{k}, y_{k}^{f}\right), v\right\rangle=(f, v)-a\left(u_{k}, v\right)-a\left(y_{k}^{f}, v\right) & \forall v \in H_{0}^{1}(\Omega), \\
\left\langle\mathcal{R}_{p}\left(y_{k}, p_{k}\right), v\right\rangle=\left(y_{k}-y^{d}, v\right)-a\left(v, p_{k}\right) & \forall v \in H_{0}^{1}(\Omega) .
\end{aligned}
$$

We note that $\mathcal{R}_{u}, \mathcal{R}_{y}$ and $\mathcal{R}_{p}$ define three sequences of uniformly bounded linear functionals in $H^{1}(\Omega)$ and $H_{0}^{1}(\Omega)$, respectively. Moreover, the orthogonality properties hold

$$
\begin{array}{rr}
\left\langle\mathcal{R}_{u}\left(u_{k}, y_{k}, p_{k}\right), v_{k}\right\rangle=0 \quad \forall v_{k} \in V_{k}, \\
\left\langle\mathcal{R}_{y}\left(u_{k}, y_{k}^{f}\right), v_{k}\right\rangle=0, \quad\left\langle\mathcal{R}_{p}\left(y_{k}, p_{k}\right), v_{k}\right\rangle=0, \quad \forall v_{k} \in V_{k}^{0} .
\end{array}
$$

Now we can show that the residuals of the control, the state and adjoint state equations in the limiting first order optimality system vanish. The proof follows from the techniques of [33, Proposition 3.1], we also refer to [20] for the related results for optimal control problems.

Lm:3.5 Lemma 4.6. Let $\mathcal{R}_{u}, \mathcal{R}_{y}$ and $\mathcal{R}_{p}$ be defined above and $\left(u_{\infty}, y_{\infty}, p_{\infty}\right)$ be the solution of the limiting control problem (4.2)-(4.3). Then there holds

$$
\begin{array}{rr}
\left\langle\mathcal{R}_{u}\left(u_{\infty}, y_{\infty}, p_{\infty}\right), v\right\rangle=0 & \forall v \in H^{1}(\Omega), \\
\left\langle\mathcal{R}_{y}\left(u_{\infty}, y_{\infty}^{f}\right), v\right\rangle=0, \quad\left\langle\mathcal{R}_{p}\left(y_{\infty}, p_{\infty}\right), v\right\rangle=0, & \forall v \in H_{0}^{1}(\Omega) .
\end{array}
$$

Proof. We only prove the vanishing property for the residuals of the control equation, the others can be proved along the same lines. We prove the result by using a density argument, so it suffices to show that $\left\langle\mathcal{R}_{u}\left(u_{\infty}, y_{\infty}, p_{\infty}\right), v\right\rangle=0$ for any $v \in H^{2}(\Omega)$.

For $k \geq l$ it is easy to see that $\mathcal{T}_{l}^{+} \subset \mathcal{T}_{k}^{+} \subset \mathcal{T}_{k}$. Therefore, we can define $\Omega_{l}^{0}=\Omega\left(\mathcal{T}_{k} \backslash \mathcal{T}_{l}^{+}\right)$and any refinement of $\mathcal{T}_{k}$ does not affect any element in $\mathcal{T}_{l}^{+}$. Let $\Pi_{k}$ be the Lagrange interpolation operator which is well-defined for the function in $H^{2}(\Omega)$. For any $v \in H^{2}(\Omega)$ with $|v|_{2, \Omega}=1$, it follows from the orthogonality property 4.32 , integration by parts and interpolation error estimate that

$$
\begin{aligned}
\left|\left\langle\mathcal{R}_{u}\left(u_{k}, y_{k}, p_{k}\right), v\right\rangle\right| & =\left|\left\langle\mathcal{R}_{u}\left(u_{k}, y_{k}, p_{k}\right), v-\Pi_{k} v\right\rangle\right| \\
& \leq C \sum_{T \in \mathcal{T}_{i}^{+}} h_{T} \eta_{u, k}\left(u_{k}, y_{k}, p_{k}, T\right)+C \sum_{T \in \mathcal{T}_{k} \mid \mathcal{T}_{l}^{+}} h_{T} \eta_{u, k}\left(u_{k}, y_{k}, p_{k}, T\right) .
\end{aligned}
$$


We see that $\left\|h_{k}\right\|_{L^{\infty}\left(\Omega_{l}^{0}\right)} \leq\left\|h_{l}\right\|_{L^{\infty}\left(\Omega_{l}^{0}\right)}$. By using the trace inequality, the inverse estimate we have

$$
\begin{aligned}
\sum_{T \in \mathcal{T}_{k} \mid \mathcal{T}_{l}^{+}} h_{T} \eta_{u, k}\left(u_{k}, y_{k}, p_{k}, T\right) & \leq C\left\|h_{k}\right\|_{L^{\infty}\left(\Omega_{l}^{0}\right)} \eta_{u, k}\left(u_{k}, y_{k}, p_{k}, \mathcal{T}_{k} \backslash \mathcal{T}_{l}^{+}\right) \\
& \leq C\left\|h_{l}\right\|_{L^{\infty}\left(\Omega_{l}^{0}\right)}\left(\left\|y^{d}-y_{k}\right\|_{0, \Omega}+\left\|\nabla u_{k}\right\|_{0, \Omega}+\left\|\nabla p_{k}\right\|_{0, \Omega}\right) \\
& \leq C\left\|h_{l}\right\|_{L^{\infty}\left(\Omega_{l}^{0}\right)}
\end{aligned}
$$

where we used the uniform boundedness of $\left\|y_{k}\right\|_{0, \Omega},\left\|u_{k}\right\|_{1, \Omega}$ and $\left\|p_{k}\right\|_{1, \Omega}$. In view of Lemma 4.4, for any given $\epsilon>0$ we may choose some sufficiently large $l$ such that

$$
\left\|h_{l}\right\|_{L^{\infty}\left(\Omega_{l}^{0}\right)} \leq \frac{\epsilon}{2 C} .
$$

On the other hand, we see that $\left\|h_{k}\right\|_{L^{\infty}\left(\Omega_{l}^{+}\right)} \lesssim 1$. Proceeding as above we have

$$
\begin{aligned}
\sum_{T \in \mathcal{T}_{l}^{+}} h_{T} \eta_{u, k}\left(u_{k}, y_{k}, p_{k}, T\right) & \leq\left\|h_{k}\right\|_{L^{\infty}\left(\Omega_{l}^{+}\right)} \eta_{u, k}\left(u_{k}, y_{k}, p_{k}, \mathcal{T}_{l}^{+}\right) \\
& \leq C \eta_{u, k}\left(u_{k}, y_{k}, p_{k}, \mathcal{T}_{l}^{+}\right) .
\end{aligned}
$$

In addition, the marking strategy 4.1 and Lemma 4.4 imply

$$
\lim _{k \rightarrow \infty} \max _{T \in \mathcal{T}_{k} \backslash \mathcal{M}_{k}} \eta_{k}\left(\left(u_{k}, y_{k}, p_{k}\right), T\right) \leq \lim _{k \rightarrow \infty} \max _{T \in \mathcal{M}_{k}} \eta_{k}\left(\left(u_{k}, y_{k}, p_{k}\right), T\right)=0,
$$

which, recalling $\mathcal{T}_{l}^{+} \cap \mathcal{M}_{k}=\emptyset$, implies

$$
\lim _{k \rightarrow \infty} \max _{T \in \mathcal{T}_{l}^{+}} \eta_{k}\left(\left(u_{k}, y_{k}, p_{k}\right), T\right)=0 .
$$

Thus, we can choose $K>l$ for some fixed $l$ such that when $k \geq K$ there holds

$$
\max _{T \in \mathcal{T}_{l}^{+}} \eta_{u, k}\left(u_{k}, y_{k}, p_{k}, T\right) \leq \max _{T \in \mathcal{T}_{l}^{+}} \eta_{k}\left(\left(u_{k}, y_{k}, p_{k}\right), T\right) \leq \frac{\epsilon}{2 C}\left|\mathcal{T}_{l}^{+}\right|^{-\frac{1}{2}} .
$$

Combining the above results we see that $\left\langle\mathcal{R}_{u}\left(u_{k}, y_{k}, p_{k}\right), v\right\rangle$ is controlled by $\epsilon$ for any $k \geq K$ and $v \in H^{2}(\Omega)$, that is to say,

$$
\left\langle\mathcal{R}_{u}\left(u_{\infty}, y_{\infty}, p_{\infty}\right), v\right\rangle=\lim _{k \rightarrow \infty}\left\langle\mathcal{R}_{u}\left(u_{k}, y_{k}, p_{k}\right), v\right\rangle=0 \quad \forall v \in H^{2}(\Omega),
$$

where we used the continuity of $\mathcal{R}_{u}$ with respect to its arguments and the convergence result in Lemma 4.3 Since $v$ is arbitrary we have $\left\langle\mathcal{R}_{u}\left(u_{\infty}, y_{\infty}, p_{\infty}\right), v\right\rangle=0$ for any $v \in H^{1}(\Omega)$. Similarly, we can prove

$$
\left\langle\mathcal{R}_{y}\left(u_{\infty}, y_{\infty}^{f}\right), v\right\rangle=0, \quad\left\langle\mathcal{R}_{p}\left(y_{\infty}, p_{\infty}\right), v\right\rangle=0, \quad \forall v \in H_{0}^{1}(\Omega) .
$$

This finishes the proof.

Furthermore, we define the following auxiliary problems: Find $\left(y^{f}\left(u_{\infty}\right), p\left(y_{\infty}\right), \tilde{u}\right) \in H_{0}^{1}(\Omega) \times H_{0}^{1}(\Omega) \times$ $H^{1}(\Omega)$ such that

$$
\left\{\begin{array}{l}
a\left(y^{f}\left(u_{\infty}\right), w\right)=(f, w)-a\left(u_{\infty}, w\right) \quad \forall w \in H_{0}^{1}(\Omega) \\
a\left(w, p\left(y_{\infty}\right)\right)=\left(y_{\infty}-y^{d}, w\right) \quad \forall w \in H_{0}^{1}(\Omega) ; \\
\alpha a(\tilde{u}, v)+(\tilde{u}, v)=a\left(v, p_{\infty}\right)+\left(y^{d}-y_{\infty}^{f}, v\right) \quad \forall v \in H^{1}(\Omega) .
\end{array}\right.
$$

It is clear that $y^{f}\left(u_{\infty}\right)=G\left(f, u_{\infty}\right)$ and $p\left(y_{\infty}\right)=G\left(y_{\infty}-y^{d}, 0\right)$. We set $y\left(u_{\infty}\right):=S\left(f, u_{\infty}\right)=y^{f}\left(u_{\infty}\right)+u_{\infty}$.

Lm:3.6 Lemma 4.7. Let $\left(u_{\infty}, y_{\infty}, p_{\infty}\right) \in H^{1}(\Omega) \times H^{1}(\Omega) \times H_{0}^{1}(\Omega)$ be the solution of the limiting control problem (4.2)- 4.3 and $\left(\tilde{u}, y\left(u_{\infty}\right), p\left(y_{\infty}\right)\right) \in H^{1}(\Omega) \times H^{1}(\Omega) \times H_{0}^{1}(\Omega)$ be the solution of the auxiliary problem 4.41 . Then there holds

$$
u_{\infty}=\tilde{u}, \quad y_{\infty}^{f}=y^{f}\left(u_{\infty}\right), \quad y_{\infty}=y\left(u_{\infty}\right), \quad p_{\infty}=p\left(y_{\infty}\right)
$$


Proof. Firstly, we can conclude from Lemma 4.5 and the third equation in 4.41) that

$$
\begin{aligned}
C\left\|u_{\infty}-\tilde{u}\right\|_{1, \Omega} & \leq \sup _{v \in H^{1}(\Omega),\|v\|_{1, \Omega}=1} \alpha a\left(\tilde{u}-u_{\infty}, v\right)+\left(\tilde{u}-u_{\infty}, v\right) \\
& =\sup _{v \in H^{1}(\Omega),\|v\|_{1, \Omega}=1}\left\langle\mathcal{R}_{u}\left(u_{\infty}, y_{\infty}, p_{\infty}\right), v\right\rangle=0,
\end{aligned}
$$

which implies the first assertion that $u_{\infty}=\tilde{u}$. Secondly, it follows from Lemma 4.6 and the first equation in (4.41) that

$$
\begin{aligned}
C\left\|y_{\infty}^{f}-y^{f}\left(u_{\infty}\right)\right\|_{1, \Omega} & \leq \sup _{v \in H_{0}^{1}(\Omega),\|v\|_{1, \Omega}=1} a\left(y^{f}\left(u_{\infty}\right)-y_{\infty}^{f}, v\right) \\
& =\sup _{v \in H_{0}^{1}(\Omega),\|v\|_{1, \Omega}=1}\left\langle\mathcal{R}_{y}\left(u_{\infty}, y_{\infty}^{f}\right), v\right\rangle=0,
\end{aligned}
$$

this proves the second claim that $y_{\infty}^{f}=y^{f}\left(u_{\infty}\right)$. Then $y_{\infty}=y\left(u_{\infty}\right)$ is a direct consequence of the first two claims. Lastly, Lemma 4.6 and the last equation in 4.41 imply

$$
\begin{aligned}
C\left\|p_{\infty}-p\left(y_{\infty}\right)\right\|_{1, \Omega} & \leq \sup _{v \in H_{0}^{1}(\Omega),\|v\|_{1, \Omega}=1} a\left(p\left(y_{\infty}\right)-p_{\infty}, v\right) \\
& =\sup _{v \in H_{0}^{1}(\Omega),\|v\|_{1, \Omega}=1}\left\langle\mathcal{R}_{p}\left(y_{\infty}, p_{\infty}\right), v\right\rangle=0,
\end{aligned}
$$

this gives $p_{\infty}=p\left(y_{\infty}\right)$. We thus completes the proof.

Now we are in the position to prove the main result of this section.

Thm:3 Theorem 4.8. Let $(u, y, p) \in H^{1}(\Omega) \times H^{1}(\Omega) \times H_{0}^{1}(\Omega)$ be the solution of optimal control problem 2.4 and $\left(u_{k}, y_{k}, p_{k}\right) \in V_{k} \times V_{k} \times V_{k}^{0}$ be the solution of the discrete problem $\sqrt{2.12}$ generated by the adaptive Algorithm 4.1] Then there hold

$$
\lim _{k \rightarrow \infty}\left\|u_{k}-u\right\|_{1, \Omega}+\left\|y_{k}-y\right\|_{1, \Omega}+\left\|p_{k}-p\right\|_{1, \Omega}=0
$$

and

$$
\lim _{k \rightarrow \infty} \eta_{k}\left(\left(u_{k}, y_{k}, p_{k}\right), \mathcal{T}_{k}\right)=0 .
$$

Proof. It follows from Theorem 3.1 that

$$
\begin{aligned}
& \lim _{k \rightarrow \infty}\left\|u_{k}-u\right\|_{1, \Omega}+\left\|y_{k}-y\right\|_{1, \Omega}+\left\|p_{k}-p\right\|_{1, \Omega} \\
\approx & \lim _{k \rightarrow \infty}\left\|u_{k}-\hat{u}\right\|_{1, \Omega}+\left\|y_{k}^{f}-y^{f}\left(u_{k}\right)\right\|_{1, \Omega}+\left\|p_{k}-p\left(y_{k}\right)\right\|_{1, \Omega} \\
= & \left\|u_{\infty}-\tilde{u}\right\|_{1, \Omega}+\left\|y_{\infty}^{f}-y^{f}\left(u_{\infty}\right)\right\|_{1, \Omega}+\left\|p_{\infty}-p\left(y_{\infty}\right)\right\|_{1, \Omega}=0,
\end{aligned}
$$

which gives the convergence of the error.

To prove the convergence of the error estimator we follow the same lines as in the proof of Lemma 4.6 to give the splitting for $k \geq l$ that

$$
\eta_{k}^{2}\left(\left(u_{k}, y_{k}, p_{k}\right), \mathcal{T}_{k}\right)=\eta_{k}^{2}\left(\left(u_{k}, y_{k}, p_{k}\right), \mathcal{T}_{l}^{+}\right)+\eta_{k}^{2}\left(\left(u_{k}, y_{k}, p_{k}\right), \mathcal{T}_{k} \backslash \mathcal{T}_{l}^{+}\right) .
$$

For the second term of the above splitting we can conclude from the lower bound in Theorem 3.5 and the local quasi-uniformity of $\mathcal{T}_{k}$ that

$$
\begin{aligned}
\eta_{k}^{2}\left(\left(u_{k}, y_{k}, p_{k}\right), \mathcal{T}_{k} \backslash \mathcal{T}_{l}^{+}\right) \leq & C\left(\left\|u_{k}-u\right\|_{1, \Omega}^{2}+\left\|y_{k}-y\right\|_{1, \Omega}^{2}+\left\|p_{k}-p\right\|_{1, \Omega}^{2}\right) \\
& +C \sum_{T \in \mathcal{T}_{k} \mid \mathcal{T}_{l}^{+}} \operatorname{osc}_{k}^{2}\left(\left(u_{k}, y_{k}, p_{k}\right), T\right) \\
\leq & C\left(\left\|u_{k}-u\right\|_{1, \Omega}^{2}+\left\|y_{k}-y\right\|_{1, \Omega}^{2}+\left\|p_{k}-p\right\|_{1, \Omega}^{2}\right) \\
& +C\left\|h_{l}\right\|_{L^{\infty}\left(\Omega_{l}^{0}\right)}^{2}\left(\|f\|_{0, \Omega}^{2}+\left\|y_{k}\right\|_{0, \Omega}^{2}+\left\|y^{d}\right\|_{0, \Omega}^{2}\right) .
\end{aligned}
$$


Since $\|f\|_{0, \Omega}^{2}+\left\|y_{k}\right\|_{0, \Omega}^{2}+\left\|y^{d}\right\|_{0, \Omega}^{2} \lesssim 1$, we are led to

$$
\begin{aligned}
\eta_{k}^{2}\left(\left(u_{k}, y_{k}, p_{k}\right), \mathcal{T}_{k}\right) \leq & \eta_{k}^{2}\left(\left(u_{k}, y_{k}, p_{k}\right), \mathcal{T}_{l}^{+}\right)+C\left\|h_{l}\right\|_{L^{\infty}\left(\Omega_{l}^{0}\right)}^{2} \\
& +C\left(\left\|u_{k}-u\right\|_{1, \Omega}^{2}+\left\|y_{k}-y\right\|_{1, \Omega}^{2}+\left\|p_{k}-p\right\|_{1, \Omega}^{2}\right) .
\end{aligned}
$$

We recall by Lemma 4.4 that $\left\|h_{l}\right\|_{L^{\infty}\left(\Omega_{l}^{0}\right)} \rightarrow 0$ as $l \rightarrow \infty$. Thus, the second term of above inequality can be made small enough by choosing $l$ large enough. For fixed $l$ we may choose sufficiently large $k \geq l$ so that $\eta_{k}^{2}\left(\left(u_{k}, y_{k}, p_{k}\right), \mathcal{T}_{l}^{+}\right)$is small, similar to the proof of Lemma 4.6. The last term can also be small if we increase $k$ further in viewing of (4.46). Therefore, for any $\epsilon>0$ we can find $k$ large enough such that $\eta_{k}\left(\left(u_{k}, y_{k}, p_{k}\right), \mathcal{T}_{k}\right) \leq \epsilon$, which implies the convergence to zero of the error estimator. This completes the proof.

\section{NUMERICAL EXPERIMENTS}

In this part, we will give some numerical examples to validate our theoretical results. In the first example we consider the case $\Omega$ is convex. We test the convergence behavior of the finite element approximations to the Dirichlet boundary control problem with quasi-uniform meshes. In the second example we consider a nonconvex domain $\Omega$. We test the efficiency and reliability of our a posteriori estimators and show the convergence of the error and estimators. All these numerical results are accordance with the theoretical predictions.

For the convenience of constructing numerical examples with exact solution, we add a priori control $u^{d}$ in the objective functional. We consider the following problem

OCP_ud

$$
\min _{u \in H^{1}(\Omega)} J(y, u)=\frac{1}{2}\left\|y-y^{d}\right\|_{0, \Omega}^{2}+\frac{\alpha}{2}\left\|\nabla\left(u-u^{d}\right)\right\|_{0, \Omega}^{2} \quad \text { subject to } \quad 1.2 .
$$

The first order optimality system is as follows: there exists $\left(u, y^{f}, p\right) \in H^{1}(\Omega) \times H_{0}^{1}(\Omega) \times H_{0}^{1}(\Omega)$ such that

OCP_OPT_ud

$$
\left\{\begin{array}{l}
a\left(y^{f}, w\right)=(f, w)-a(u, w) \quad \forall w \in H_{0}^{1}(\Omega) ; \\
a(w, p)=\left(y-y^{d}, w\right) \quad \forall w \in H_{0}^{1}(\Omega) ; \\
\alpha a(u, v)=a(v, p)+\alpha a\left(u^{d}, v\right)+\left(y^{d}-y, v\right) \quad \forall v \in H^{1}(\Omega),
\end{array}\right.
$$

where $y=y^{f}+u \in H^{1}(\Omega)$.

Suppose that $\Delta u^{d} \in L^{2}(\Omega)$ and we define

$$
\begin{aligned}
\eta_{u, h}\left(u_{h}, y_{h}, p_{h}, T\right):= & \left(h_{T}^{2}\left\|y^{d}-y_{h}+\alpha \Delta u^{d}\right\|_{0, T}^{2}+\sum_{E \in \mathcal{E}_{h}^{h}, E \subset \partial T} h_{E}\left\|\nabla\left(\alpha\left(u_{h}-u^{d}\right)-p_{h}\right) \cdot n_{E}\right\|_{0, E}^{2}\right. \\
& \left.+\sum_{E \in \mathcal{E}_{h}^{i}, E \subset \partial T} h_{E}\left\|\left[\nabla\left(\left(\alpha u_{h}-u^{d}\right)-p_{h}\right) \cdot n_{E}\right]\right\|_{0, E}^{2}\right)^{\frac{1}{2}} .
\end{aligned}
$$

Then all the results in previous sections hold with the similar analysis.

We denote the $L^{2}$-norm error, the $H^{1}$-norm error and the values of the estimators by $e_{0, h}=\left\|u-u_{h}\right\|_{0}+$ $\left\|y-y_{h}\right\|_{0}+\left\|p-p_{h}\right\|_{0}, e_{1, h}=\left\|u-u_{h}\right\|_{1}+\left\|y-y_{h}\right\|_{1}+\left\|p-p_{h}\right\|_{1}$ and $\eta_{N}$, respectively.

Exm:1 Example 5.1. Let $\Omega=(0,1)^{2}$. We choose the data

$$
\begin{array}{r}
y_{d}=\sin \left(k_{1} \pi x_{1}\right) \sin \left(k_{1} \pi x_{2}\right)+2 k_{2}^{2} \pi^{2}\left[\cos \left(2 k_{2} \pi x_{1}\right) \sin ^{2}\left(k_{2} \pi x_{2}\right)+\sin ^{2}\left(k_{2} \pi x_{1}\right) \cos \left(2 k_{2} \pi x_{2}\right)\right], \\
f=2 k_{1}^{2} \pi^{2} \sin \left(k_{1} \pi x_{1}\right) \sin \left(k_{1} \pi x_{2}\right), \quad u^{d}=\sin \left(k_{1} \pi x_{1}\right) \sin \left(k_{1} \pi x_{2}\right),
\end{array}
$$

where $k_{1}, k_{2}$ are positive integers. Then for any $\alpha>0$, the exact solutions are

$$
u=\sin \left(k_{1} \pi x_{1}\right) \sin \left(k_{1} \pi x_{2}\right), \quad y=\sin \left(k_{1} \pi x_{1}\right) \sin \left(k_{1} \pi x_{2}\right), \quad p=\sin ^{2}\left(k_{2} \pi x_{1}\right) \sin ^{2}\left(k_{2} \pi x_{2}\right) .
$$

In our numerical test we take $\alpha=1, k_{1}=k_{2}=1$. The mesh is refined uniformly to test a priori error estimate. The $L^{2}$-norm error, $H^{1}$-norm error and the orders of convergence with respect to the mesh size are listed in Table 1 . Figure 1 shows the convergence rate with slope. According to these results, we know that the orders of convergence of $L^{2}$-norm and $H^{1}$-norm errors are 2 and 1, respectively, which agrees with the theoretical analysis in [6] and current paper. 
TABLE 1. $L^{2}$-norm and $H^{1}$-norm errors versus mesh size $h$ and orders of convergence for Example 5.1.

\begin{tabular}{||c|c|c|c|c|}
\hline$h$ & $e_{0, h}$ & order & $e_{1, h}$ & order \\
\hline $1 / 4$ & $7.7012 \mathrm{e}-2$ & - & 1.6266 & - \\
\hline $1 / 8$ & $2.0971 \mathrm{e}-2$ & 1.8767 & $8.4052 \mathrm{e}-1$ & 0.9525 \\
\hline $1 / 16$ & $5.4192 \mathrm{e}-3$ & 1.9523 & $4.2558 \mathrm{e}-1$ & 0.9819 \\
\hline $1 / 32$ & $1.3701 \mathrm{e}-3$ & 1.9838 & $2.1367 \mathrm{e}-1$ & 0.9941 \\
\hline $1 / 64$ & $3.4374 \mathrm{e}-4$ & 1.9949 & $1.0697 \mathrm{e}-1$ & 0.9982 \\
\hline $1 / 128$ & $8.6030 \mathrm{e}-5$ & 1.9984 & $5.3503 \mathrm{e}-2$ & 0.9995 \\
\hline $1 / 256$ & $2.1515 \mathrm{e}-5$ & 1.9995 & $2.6755 \mathrm{e}-2$ & 0.9998 \\
\hline
\end{tabular}

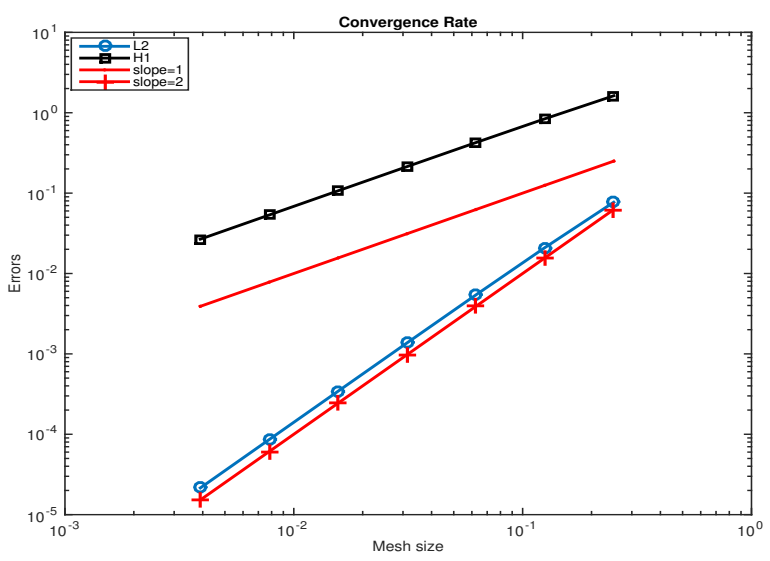

Figure 1. The convergence rate on uniformly refined meshes for Example 5.1.

Exm:2 Example 5.2. Let $\Omega=(-1,1)^{2} \backslash([0,1] \times[-1,0])$ be a L-shaped domain which will be shown in Figure 3 . Set $y_{d}=r^{\frac{2}{3}} \sin \left(\frac{2}{3} \theta\right)+2 k^{2} \pi^{2}\left[\cos \left(2 k \pi x_{1}\right) \sin ^{2}\left(k \pi x_{2}\right)+\sin ^{2}\left(k \pi x_{1}\right) \cos \left(2 k \pi x_{2}\right)\right], f=0, u_{d}=r^{\frac{2}{3}} \sin \left(\frac{2}{3} \theta\right)$, where $k$ is a positive integer and $(r, \theta)$ corresponds to the polar coordinates. Then for any $\alpha>0$, the exact solution is $u=r^{\frac{2}{3}} \sin \left(\frac{2}{3} \theta\right), y=r^{\frac{2}{3}} \sin \left(\frac{2}{3} \theta\right)$ and $p=\sin ^{2}\left(k \pi x_{1}\right) \sin ^{2}\left(k \pi x_{2}\right)$.

In this numerical test we choose $\alpha=1$ and $k=1$. We adopt Dörfler's strategy for the MARK procedure and the newest vertex bisection algorithm for the mesh refinements. The $H^{1}$-norm error, the values of the estimators and the reduction rates of the $H^{1}$-norm error and the estimator with respect to degrees of freedom (denoted by $N$ ) of the finite element space are listed in Table 2 . The reduction rate is shown in Figure 2 and Figure 3 gives the adaptively refined mesh. As shown in these results, the reduction rate of the $H^{1}$ norm error and the estimator is approximately $N^{-1 / 2}$, which is the optimal rate we can expect with linear finite elements. We can observe from the refined mesh shown in Figure 3 that the estimator can capture the singularity of the solutions. These results validate the efficiency and reliability of our a posteriori estimator and indicate, to some extent, the convergence of the estimator to 0 and the solution to the exact solution as the adaptive loops increase, just as we expected from the theoretical analysis.

Acknowledgements: The first author was supported by the National Natural Science Foundation of China under grants 11671391 and 91530204 . The third author acknowledged the support of the National Natural Science Foundation of China under grants 11571356 and 91530204. 
TABLE 2. The $H^{1}$-norm error and the values of estimators versus DOFs $N$ and orders of convergence for Example 5.2

\begin{tabular}{||c|c|c|c|c|}
\hline$N$ & $e_{1, h}$ & order & $\eta_{N}$ & order \\
\hline 65 & 2.3129 & - & 11.0076 & - \\
\hline 85 & 1.9416 & -0.6524 & 9.4324 & -0.5757 \\
\hline 126 & 1.6631 & -0.3933 & 7.8971 & -0.4513 \\
\hline 181 & 1.2766 & -0.7302 & 6.6603 & -0.4703 \\
\hline 252 & 1.1040 & -0.4388 & 5.7615 & -0.4380 \\
\hline 353 & $9.4964 \mathrm{e}-1$ & -0.4533 & 4.9560 & -0.4468 \\
\hline 517 & $7.5554 \mathrm{e}-1$ & -0.5937 & 4.0198 & -0.5487 \\
\hline 764 & $6.3204 \mathrm{e}-1$ & -0.4570 & 3.3759 & -0.4470 \\
\hline 1072 & $5.5274 \mathrm{e}-1$ & -0.3958 & 2.9323 & -0.4160 \\
\hline 1573 & $4.3215 \mathrm{e}-1$ & -0.6418 & 2.3350 & -0.5940 \\
\hline 2418 & $3.5046 \mathrm{e}-1$ & -0.4873 & 1.9085 & -0.4691 \\
\hline 3582 & $2.9539 \mathrm{e}-1$ & -0.4351 & 1.5964 & -0.4544 \\
\hline 5481 & $2.3451 \mathrm{e}-1$ & -0.5425 & 1.2826 & -0.5145 \\
\hline
\end{tabular}

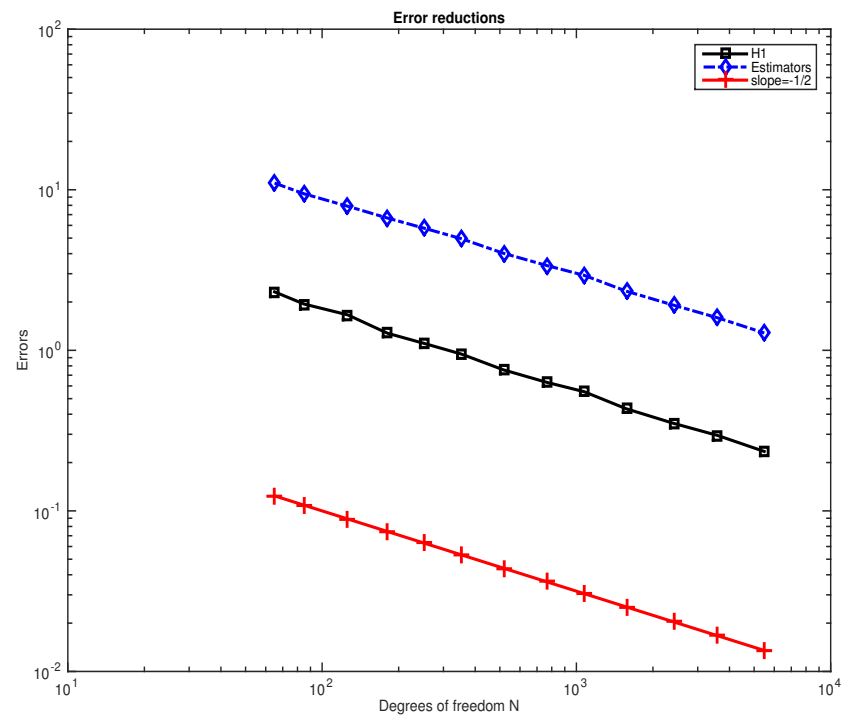

FigURE 2. The reduction rate of the $H^{1}$-norm errors and error estimators on adaptively refined meshes for Example 5.2 .

Appendix A. Proof of Theorem 2.3

We intend to derive a priori error estimates by following the standard approach of introducing some auxiliary approximations. To begin with, we introduce the following problems: Find $\left(y_{h}^{f}(u), p_{h}(y), \tilde{u}_{h}\right) \in$ $V_{h}^{0} \times V_{h}^{0} \times V_{h}$ such that

$$
\left\{\begin{array}{l}
a\left(y_{h}^{f}(u), w_{h}\right)=\left(f, w_{h}\right)-a\left(u, w_{h}\right) \quad \forall w_{h} \in V_{h}^{0} \\
a\left(w_{h}, p_{h}(y)\right)=\left(y-y^{d}, w_{h}\right) \quad \forall w_{h} \in V_{h}^{0} \\
\alpha a\left(\tilde{u}_{h}, v_{h}\right)+\left(\tilde{u}_{h}, v_{h}\right)=a\left(v_{h}, p\right)+\left(y^{d}-y^{f}, v_{h}\right) \quad \forall v_{h} \in V_{h} .
\end{array}\right.
$$




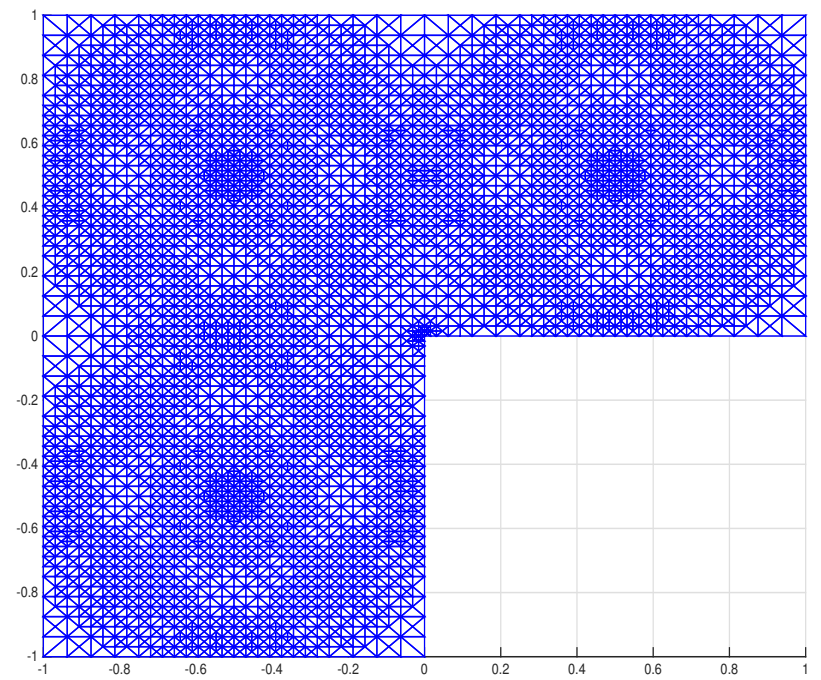

FIGURE 3. Adaptively refined mesh after 13 iteratios for Example 5.2 .

Moreover, we define $y_{h}^{f}\left(\tilde{u}_{h}\right) \in V_{h}^{0}$ such that

$$
a\left(y_{h}^{f}\left(\tilde{u}_{h}\right), w_{h}\right)=\left(f, w_{h}\right)-a\left(\tilde{u}_{h}, w_{h}\right) \quad \forall w_{h} \in V_{h}^{0} .
$$

We set $y_{h}(u):=S_{h}(f, u)=y_{h}^{f}(u)+u$ and $y_{h}\left(\tilde{u}_{h}\right):=S_{h}\left(f, \tilde{u}_{h}\right)=y_{h}^{f}\left(\tilde{u}_{h}\right)+\tilde{u}_{h}$. It is clear that $y_{h}^{f}(u)$ and $p_{h}(y)$ are the finite element approximations of $y^{f}$ and $p$ in $V_{h}^{0}$, respectively. Moreover, $\tilde{u}_{h}$ is the finite element approximation of $u$ in $V_{h}$ in the sense of 2.8.

Lm:0 Lemma A.1. Let $(y, p, u) \in H^{1}(\Omega) \times H_{0}^{1}(\Omega) \times H^{1}(\Omega)$ be the solution of the optimal control problem (2.4) and $\left(y^{f}\left(u_{h}\right), p\left(y_{h}\right), \hat{u}\right) \in H_{0}^{1}(\Omega) \times H_{0}^{1}(\Omega) \times H^{1}(\Omega)$ be the solution of the auxiliary problems $\sqrt[A .1]{ }$. Then we have

$$
\begin{aligned}
& \left\|u-u_{h}\right\|_{1, \Omega}+\left\|y-y_{h}\right\|_{1, \Omega}+\left\|p-p_{h}\right\|_{1, \Omega} \\
\lesssim & \left\|u-\tilde{u}_{h}\right\|_{1, \Omega}+\left\|y^{f}-y_{h}^{f}\right\|_{1, \Omega}+\left\|p-p_{h}(y)\right\|_{1, \Omega} .
\end{aligned}
$$

Proof. From 2.12, A.1, and A.2 we have

\begin{tabular}{|l|}
\hline 1_est_2 \\
\hline 1_est_3 \\
\hline 1_est_4 \\
\hline
\end{tabular}

$$
\begin{array}{r}
a\left(y_{h}^{f}-y_{h}^{f}\left(\tilde{u}_{h}\right), w_{h}\right)=a\left(\tilde{u}_{h}-u_{h}, w_{h}\right) \quad \forall w_{h} \in V_{h}^{0} ; \\
a\left(w_{h}, p_{h}(y)-p_{h}\right)=\left(y-y_{h}, w_{h}\right) \quad \forall w_{h} \in V_{h}^{0} ; \\
\alpha a\left(u_{h}-\tilde{u}_{h}, v_{h}\right)+\left(u_{h}-\tilde{u}_{h}, v_{h}\right)=a\left(v_{h}, p_{h}-p\right)+\left(y^{f}-y_{h}^{f}, v_{h}\right) \quad \forall v_{h} \in V_{h} .
\end{array}
$$

Setting $w_{h}=p_{h}(y)-p_{h}$ in A.4 and $w_{h}=y_{h}^{f}-y_{h}^{f}\left(\tilde{u}_{h}\right)$ in A.5 we are led to

$$
a\left(\tilde{u}_{h}-u_{h}, p_{h}(y)-p_{h}\right)=\left(y-y_{h}, y_{h}^{f}-y_{h}^{f}\left(\tilde{u}_{h}\right)\right) .
$$


From the triangle inequality it suffices to prove $\left\|u_{h}-\tilde{u}_{h}\right\|_{1, \Omega}$. We can derive by setting $v=u_{h}-\tilde{u}_{h}$ in A.6) that

$$
\begin{aligned}
\alpha\left\|\nabla\left(u_{h}-\tilde{u}_{h}\right)\right\|_{0, \Omega}^{2}= & a\left(u_{h}-\tilde{u}_{h}, p_{h}-p\right)+\left(y^{f}-y_{h}^{f}, u_{h}-\tilde{u}_{h}\right)-\left(u_{h}-\tilde{u}_{h}, u_{h}-\tilde{u}_{h}\right) \\
= & a\left(u_{h}-\tilde{u}_{h}, p_{h}-p_{h}(y)\right)+a\left(u_{h}-\tilde{u}_{h}, p_{h}(y)-p\right)+\left(y^{f}-y_{h}^{f}, u_{h}-\tilde{u}_{h}\right) \\
& -\left(u_{h}-\tilde{u}_{h}, u_{h}-\tilde{u}_{h}\right)+a\left(u_{h}-\tilde{u}_{h}, p_{h}(y)-p_{h}\right)+\left(y-y_{h}, y_{h}^{f}-y_{h}^{f}\left(\tilde{u}_{h}\right)\right) \\
= & a\left(u_{h}-\tilde{u}_{h}, p_{h}(y)-p\right)+\left(y^{f}-y_{h}^{f}, u_{h}-\tilde{u}_{h}\right) \\
& +\left(u_{h}-\tilde{u}_{h}, \tilde{u}_{h}-u_{h}\right)+\left(y-y_{h}, y_{h}^{f}-y_{h}^{f}\left(\tilde{u}_{h}\right)\right) .
\end{aligned}
$$

Note that

$$
\begin{aligned}
& \left(y^{f}-y_{h}^{f}, u_{h}-\tilde{u}_{h}\right)+\left(u_{h}-\tilde{u}_{h}, \tilde{u}_{h}-u_{h}\right)+\left(y-y_{h}, y_{h}^{f}-y_{h}^{f}\left(\tilde{u}_{h}\right)\right) \\
= & \left(y^{f}-y_{h}^{f}, u_{h}-\tilde{u}_{h}\right)+\left(u_{h}-\tilde{u}_{h}, \tilde{u}_{h}-u_{h}\right)+\left(y-y_{h}, y_{h}-y_{h}\left(\tilde{u}_{h}\right)\right)+\left(y-y_{h}, \tilde{u}_{h}-u_{h}\right) \\
= & \left(u_{h}-u, u_{h}-\tilde{u}_{h}\right)+\left(u_{h}-\tilde{u}_{h}, \tilde{u}_{h}-u_{h}\right)+\left(y-y_{h}\left(\tilde{u}_{h}\right), y_{h}-y_{h}\left(\tilde{u}_{h}\right)\right)+\left(y_{h}\left(\tilde{u}_{h}\right)-y_{h}, y_{h}-y_{h}\left(\tilde{u}_{h}\right)\right) \\
= & -\left\|y_{h}-y_{h}\left(\tilde{u}_{h}\right)\right\|_{0, \Omega}^{2}+\left(\tilde{u}_{h}-u, u_{h}-\tilde{u}_{h}\right)+\left(y-y_{h}\left(\tilde{u}_{h}\right), y_{h}-y_{h}\left(\tilde{u}_{h}\right)\right) .
\end{aligned}
$$

Therefore, we are led to

$$
\begin{aligned}
& \alpha\left\|\nabla\left(u_{h}-\tilde{u}_{h}\right)\right\|_{0, \Omega}^{2}+\left\|y_{h}-y_{h}\left(\tilde{u}_{h}\right)\right\|_{0, \Omega}^{2} \\
= & a\left(u_{h}-\tilde{u}_{h}, p_{h}(y)-p\right)+\left(\tilde{u}_{h}-u, u_{h}-\tilde{u}_{h}\right)+\left(y-y_{h}\left(\tilde{u}_{h}\right), y_{h}-y_{h}\left(\tilde{u}_{h}\right)\right) .
\end{aligned}
$$

Furthermore, we can derive

$$
\begin{aligned}
\left\|y-y_{h}\left(\tilde{u}_{h}\right)\right\|_{0, \Omega} & =\left\|G(f, u)+u-G_{h}\left(f, \tilde{u}_{h}\right)-\tilde{u}_{h}\right\|_{0, \Omega} \\
& \leq C\left(\left\|\tilde{u}_{h}-u\right\|_{0, \Omega}+\left\|G(f, u)-G_{h}(f, u)\right\|_{0, \Omega}+\left\|G_{h}(f, u)-G_{h}\left(f, \tilde{u}_{h}\right)\right\|_{0, \Omega}\right) \\
& \leq C\left(\left\|\tilde{u}_{h}-u\right\|_{0, \Omega}+\left\|y^{f}-y_{h}^{f}(u)\right\|_{1, \Omega}+\left\|\nabla\left(\tilde{u}_{h}-u\right)\right\|_{0, \Omega}\right) .
\end{aligned}
$$

We can conclude from Lemma 2.2 that $\left\|y_{h}-y_{h}\left(\tilde{u}_{h}\right)\right\|_{0, \Omega}^{2}+\alpha\left\|\nabla\left(u_{h}-\tilde{u}_{h}\right)\right\|_{0, \Omega}^{2} \approx\left\|u_{h}-\tilde{u}_{h}\right\|_{1, \Omega}^{2}$. Therefore, Cauchy-Schwarz and Young's inequalities give

$$
\begin{aligned}
& \alpha\left\|\nabla\left(u_{h}-\tilde{u}_{h}\right)\right\|_{0, \Omega}^{2}+\left\|y_{h}-y_{h}\left(\tilde{u}_{h}\right)\right\|_{0, \Omega}^{2} \\
\lesssim & \left\|\nabla\left(u-\tilde{u}_{h}\right)\right\|_{0, \Omega}^{2}+\left\|p-p_{h}(y)\right\|_{1, \Omega}^{2}+\left\|y^{f}-y_{h}^{f}(u)\right\|_{1, \Omega}^{2}+\left\|u-\tilde{u}_{h}\right\|_{0, \Omega}^{2} .
\end{aligned}
$$

Since $u$ is harmonic, we see $y_{h}^{f}=y_{h}^{f}(u)$. Thus, we arrive at

$$
\left\|u_{h}-\tilde{u}_{h}\right\|_{1, \Omega}^{2} \lesssim\left\|u-\tilde{u}_{h}\right\|_{1, \Omega}^{2}+\left\|p-p_{h}(y)\right\|_{1, \Omega}^{2}+\left\|y^{f}-y_{h}^{f}\right\|_{1, \Omega}^{2} .
$$

Note that $y_{h}\left(\tilde{u}_{h}\right)-y_{h}=\tilde{u}_{h}-u_{h}+G_{h}\left(f, \tilde{u}_{h}\right)-G_{h}\left(f, u_{h}\right)$ and $y-y_{h}\left(\tilde{u}_{h}\right)=u-\tilde{u}_{h}+G(f, u)-G_{h}\left(f, \tilde{u}_{h}\right)$. It is not difficult to prove

$$
\begin{aligned}
\left\|y-y_{h}\right\|_{1, \Omega} & \lesssim\left\|y-y_{h}\left(\tilde{u}_{h}\right)\right\|_{1, \Omega}+\left\|y_{h}\left(\tilde{u}_{h}\right)-y_{h}\right\|_{1, \Omega} \\
& \lesssim\left\|u-\tilde{u}_{h}\right\|_{1, \Omega}+\left\|G(f, u)-G_{h}\left(f, \tilde{u}_{h}\right)\right\|_{1, \Omega}+\left\|\tilde{u}_{h}-u_{h}\right\|_{1, \Omega}+\left\|G_{h}\left(f, \tilde{u}_{h}\right)-G_{h}\left(f, u_{h}\right)\right\|_{1, \Omega} \\
& \lesssim\left\|u-\tilde{u}_{h}\right\|_{1, \Omega}+\left\|\tilde{u}_{h}-u_{h}\right\|_{1, \Omega}+\left\|G(f, u)-G_{h}(f, u)\right\|_{1, \Omega}+\left\|G_{h}(f, u)-G_{h}\left(f, \tilde{u}_{h}\right)\right\|_{1, \Omega} \\
& \lesssim\left\|u-\tilde{u}_{h}\right\|_{1, \Omega}+\left\|\tilde{u}_{h}-u_{h}\right\|_{1, \Omega}+\left\|y^{f}-y_{h}^{f}\right\|_{1, \Omega}
\end{aligned}
$$

and

$$
\left\|p_{h}(y)-p_{h}\right\|_{1, \Omega} \lesssim\left\|y-y_{h}\right\|_{0, \Omega} .
$$

We thus complete the proof of A.3 by collecting the above results.

Proof of Theorem 2.3. Since $y_{h}^{f}(u)$ and $p_{h}(y)$ are the finite element approximations of $y^{f}$ and $p$ in $V_{h}^{0}, \tilde{u}_{h}$ is the finite element approximation of $u$ in $V_{h}$ in the sense of 2.8. From A.3. and standard a priori error estimate for elliptic equation we have

$$
\left\|u-u_{h}\right\|_{1, \Omega}+\left\|y-y_{h}\right\|_{1, \Omega}+\left\|p-p_{h}\right\|_{1, \Omega} \leq C h\left(\|u\|_{2, \Omega}+\|y\|_{2, \Omega}+\|p\|_{2, \Omega}\right) .
$$


Moreover, it follows from [6, Lemma 2.5] that

$$
\|u\|_{2, \Omega}+\|y\|_{2, \Omega}+\|p\|_{2, \Omega} \leq C\left(\|f\|_{0, \Omega}+\left\|y^{d}\right\|_{0, \Omega}\right) .
$$

We thus complete the proof of 2.20 .

\section{REFERENCES}

Apel

Babuska

abuska_Vogelius

Casas

Casas_ESAIM

Chowdhury

Ciarlet

Clement

Hinze

Dorfler

Gaevskaya

Gong_Hinze_Zhou

Gong_Yan_SICON

Gong_Yan

Gong_2016

Gong

Hoppe 1

Hinze09book

Kohls

Kohls1

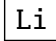

Lions

Liu2

Liu5

Liu3

LiuYan08book

Mateos

May

Morin

[1] T. Apel, M. Mateos, J. Pfefferer and A. Rösch, On the regularity of the solutions of Dirichlet optimal control problems in polygonal domains, SIAM J. Control Optim., 53 (2015), no. 6, pp. 3620-3641.

[2] I. Babuška and W.C. Rheinboldt, Error estimates for adaptive finite element computations, SIAM J. Numer. Anal., 15 (1978), pp. 736-754.

[3] I. Babuška and M. Vogelius, Feedback and adaptive finite element solution of one-dimensional boundary value problems, Numer. Math., 44 (1984), pp. 75-102.

[4] E. Casas, J. P. Raymond, Error estimates for the numerical approximation of Dirichlet boundary control for semilinear elliptic equations, SIAM J. Control Optim., 45 (2006), pp. 1586-1611.

[5] E. Casas, M. Mateos and J.P. Raymond, Penalization of Dirichlet optimal control problems, ESAIM Control Optim. Calc. Var., 15 (2009), no. 4, pp. 782-809.

[6] S. Chowdhury, T. Gudi and A.K. Nandakumaran, Error bounds for a Dirichlet boundary control problem based on energy spaces, Math. Comp., 86 (2017), no. 305, pp. 1103-1126.

[7] P.G. Ciarlet, The Finite Element Methods for Elliptic Problems, North-Holland, Amsterdam, 1978.

[8] Ph. Clément, Approximation by finite element functions using local regularization, RAIRO Anal. Numer., 9 (1975), pp. 77-84.

[9] K. Deckelnick, A. Günther, M. Hinze, Finite element approximation of Dirichlet boundary control for elliptic PDEs on two and three-dimensional curved domains. SIAM J. Control Optim., 48(2009), pp. 2798-2819.

[10] W. Dörfler, A convergent adaptive algorithm for Poisson's equation, SIAM J. Numer. Anal., 33 (1996), pp. 1106-1124.

[11] A. Gaevskaya, R.H.W. Hoppe, Y. Iliash and M. Kieweg, Convergence analysis of an adaptive finite element method for distributed control problems with control constraints, in Control of coupled partial differential equations, vol. 155 of Internat. Ser. Numer. Math., Birkhäuser, Basel, 2007, pp. 47-68.

[12] W. Gong, M. Hinze and Z.J. Zhou, Finite element method and a priori error estimates for Dirichlet boundary control problems governed by parabolic PDEs, J. Sci. Comput., 66 (2016), no. 3, pp. 941-967.

[13] W. Gong and N.N. Yan, Mixed finite element method for Dirichlet boundary control problem governed by elliptic PDEs, SIAM J. Control Optim., 49 (2011), pp. 984-1014.

[14] W. Gong and N.N. Yan, Adaptive finite element method for elliptic optimal control problems: convergence and optimality, Numer. Math., 135 (2017), no. 4, pp. 1121-1170

[15] W. Gong, N.N. Yan and Z.J. Zhou, Convergence of $L^{2}$-norm based adaptive finite element method for elliptic optimal control problems, arXiv:1608.08699, 2016.

[16] W. Gong, H.H. Xie and N.N. Yan, Adaptive multilevel correction method for finite element approximations of elliptic optimal control problems, J. Sci. Comput., 72 (2017), no. 2, pp. 820-841.

[17] M. Hintermüller, R.H.W. Hoppe, Y. Iliash and M. Kieweg, An a posteriori error analysis of adaptive finite element methods for distributed elliptic control problems with control constraints, ESAIM: Control Optim. Calc. Var., 14 (2008), pp. 540-560.

[18] M. Hinze, R. Pinnau, M. Ulbrich and S. Ulbrich, Optimization with PDE Constraints, Math. Model. Theo. Appl., 23, Springer, New York, 2009.

[19] K. Kohls, A. Rösch and K.G. Siebert, A posteriori error analysis of optimal control problems with control constraints, SIAM J. Control Optim., 52 (2014), pp. 1832-1861.

[20] K. Kohls, A. Rösch and K.G. Siebert, Convergence of adaptive finite elements for optimal control problems with control constraints, G. Leugering et al. (eds.), Trends in PDE Constrained Optimization, vol. 165 of Internat. Ser. Numer. Math., 2015, pp. 403-419.

[21] R. Li, W.B. Liu, H.P. Ma and T. Tang, Adaptive finite element approximation for distributed elliptic optimal control problems, SIAM J. Control Optim., 41 (2002), no. 5, pp. 1321-1349.

[22] J.L. Lions, Optimal Control of Systems Governed by Partial Differential Equations, Springer-Verlag, Berlin, 1971.

[23] W.B. Liu and N.N. Yan, A posteriori error analysis for convex distributed optimal control problems, Adv. Comp. Math., 15 (2001), no. 1-4, pp. 285-309.

[24] W.B. Liu and N.N. Yan, A posteriori error estimates for convex boundary control problems, SIAM J. Numer. Anal., 39 (2001), no. 1, pp. 73-99.

[25] W.B. Liu and N.N. Yan, A posteriori error estimates for optimal problems governed by Stokes equations, SIAM J. Numer. Anal., 40 (2003), pp. 1850-1869.

[26] W.B. Liu and N.N. Yan, Adaptive Finite Element Methods for Optimal Control Governed by PDEs, Science press, Beijing, 2008.

[27] M. Mateos, Optimization methods for Dirichlet control problems, arXiv:1701.07619, 2017.

[28] S. May, R. Rannacher and B. Vexler, Error analysis for a finite element approximation of elliptic Dirichlet boundary control problems. SIAM J. Control Optim., 51 (2013), no. 3, pp. 2585-2611.

[29] P. Morin, R.H. Nochetto and K.G. Siebert, Data oscillation and convergence of adaptive FEM, SIAM J. Numer. Anal., 38 (2000), pp. 466-488. 
Siebert_Veeser

of

Scott

Siebert

Xu_Zou_MCOM

Xu_Zou_SICON

Verfuth
[30] P. Morin, K.G. Siebert and A. Veeser, A basic convergence result for conforming adaptive finite elements, Math. Models Methods Appl. Sci., 18 (2008), pp. 707-737.

[31] G. Of, T.X. Phan and O. Steinbach, An energy space finite element approach for elliptic Dirichlet boundary control problems, Numer. Math., 129 (2015), no. 4, pp. 723-748.

[32] L.R. Scott and S. Zhang, Finite element interpolation of nonsmooth functions satisfying boundary conditions, Math. Comput., 54 (1990), pp. 483-493.

[33] K.G. Siebert, A convergence proof for adaptive finite elements without lower bound, IMA J. Numer. Anal., 31 (2011), pp. 947-970.

[34] Y.F. Xu and J. Zou, Convergence of an adaptive finite element method for distributed flux reconstruction, Math. Comp., 84 (2015), pp. 2645-2663.

[35] Y.F. Xu and J. Zou, Analysis of an adaptive finite element method for recovering the Robin coefficient, SIAM J. Control Optim., 53 (2015), no. 2, pp. 622-644.

[36] R. Verfürth, A Review of a Posteriori Error Estimates and Adaptive Mesh Refinement Techniques, Wiley-Teubner, New York, 1996. 